\title{
On a Stochastic Camassa-Holm Type Equation with Higher Order Nonlinearities
}

\author{
Christian Rohde ${ }^{1} \cdot$ Hao Tang ${ }^{1}$
}

Received: 8 January 2020 / Revised: 5 May 2020 / Published online: 27 July 2020

(c) The Author(s) 2020

\begin{abstract}
The subject of this paper is a generalized Camassa-Holm equation under random perturbation. We first establish local existence and uniqueness results as well as blow-up criteria for pathwise solutions in the Sobolev spaces $H^{s}$ with $s>3 / 2$. Then we analyze how noise affects the dependence of solutions on initial data. Even though the noise has some already known regularization effects, much less is known concerning the dependence on initial data. As a new concept we introduce the notion of stability of exiting times and construct an example showing that multiplicative noise (in Itô sense) cannot improve the stability of the exiting time, and simultaneously improve the continuity of the dependence on initial data. Finally, we obtain global existence theorems and estimate associated probabilities.
\end{abstract}

Keywords Stochastic generalized Camassa-Holm equation · Pathwise solution · Noise effect $\cdot$ Exiting time $\cdot$ Dependence on initial data $\cdot$ Global existence

Mathematics Subject Classification Primary: 60H15 - 35Q51; Secondary: 35A01 $\cdot 35 B 30$

\section{Introduction}

We consider a stochastic version of the generalized Camassa-Holm equation. Let $t$ denote the time variable and let $x$ be the one-dimensional space variable. The equation is given for $k \in \mathbb{N}$ by

$$
u_{t}-u_{x x t}+(k+2) u^{k} u_{x}-\left(1-\partial_{x x}^{2}\right) h(t, u) \dot{\mathcal{W}}=(k+1) u^{k-1} u_{x} u_{x x}+u^{k} u_{x x x} .
$$

C. R. acknowledges funding by Deutsche Forschungsgemeinschaft (DFG, German Research Foundation) under Germany's Excellence Strategy_EXC 2075-390740016. H. T. is supported by the Alexander von Humboldt Foundation.

$凶 \quad$ Hao Tang

Hao.Tang@mathematik.uni-stuttgart.de

Christian Rohde

christian.rohde@mathematik.uni-stuttgart.de

1 Institut für Angewandte Analysis und Numerische Simulation, Universität Stuttgart, Pfaffenwaldring 57, 70569 Stuttgart, Germany 
In (1.1), $h: \mathbb{R}^{+} \times \mathbb{R} \rightarrow \mathbb{R}$ is some nonlinear function and $\dot{\mathcal{W}}$ is a cylindrical Wiener process. We will consider (1.1) on the torus, i.e. $x \in \mathbb{T}=\mathbb{R} / 2 \pi \mathbb{Z}$.

For $h=0$ and $k=1$, Eq. (1.1) reduces to the deterministic Camassa-Holm (CH) equation given by

$$
u_{t}-u_{x x t}+3 u u_{x}=2 u_{x} u_{x x}+u u_{x x x} .
$$

Fokas \& Fuchssteiner [24] introduced (1.2) when studying completely integrable generalizations of the Korteweg-de-Vries (KdV) equation with bi-Hamiltonian structure whereas Camassa \& Holm [8] proposed (1.2) to describe the unidirectional propagation of shallow water waves over a flat bottom. Since then, the CH equation (1.2) has been studied intensively, and we cannot even attempt to survey the vast research history here. For the paper at hand it is important to mention the wave-breaking phenomenon which illustrates possible loss of regularity as a fundamental mechanism in the $\mathrm{CH}$ equations. In contrast to smooth soliton solutions to the $\mathrm{KdV}$ equation [44], solutions to the $\mathrm{CH}$ equation remain indeed bounded but their slope can become unbounded in finite time, cf. [14] and related work in [12,15,52]. Moreover, for a smooth initial profile, it is possible to predict exactly (by establishing a necessary and sufficient condition) whether wave-breaking occurs for solutions to the Cauchy problem for $(1.2)$ [14,52]. The other essential feature of the $\mathrm{CH}$ equation is the occurrence of traveling waves with a peak at their crest, exactly like that the governing equations for water waves admit the so-called Stokes waves of the greatest height, see $[13,16,17]$. Bressan $\&$ Constantin proved the existence of dissipative as well as conservative solutions in [6,7]. Later, Holden \& Raynaud [37,38] also obtained global conservative and dissipative solutions using Lagrangian transport ideas.

When $h=0$ and $k=2$, Eq. (1.1) forms the cubic equation

$$
u_{t}-u_{x x t}+4 u^{2} u_{x}=3 u u_{x} u_{x x}+u^{2} u_{x x x}
$$

which has been derived by Novikov in [55]. It has been proven that (1.3) possesses a biHamiltonian structure with an infinite sequence of conserved quantities and (1.3) admits peaked solutions of explicit form $u(t, x)= \pm \sqrt{c} e^{-|x-c t|}$ with $c>0$ [26], as well as multipeakon solutions with explicit formulas [39]. For the study of other deterministic instances of $(1.1)$ we refer to $[9,32,66,67]$.

Equations like (1.2) are naturally embedded in high-order descriptions with energydissipative evolution. A weakly dissipative evolution is given for some parameter $\lambda>0$ by

$u_{t}-u_{x x t}+(k+2) u^{k} u_{x}-\left(1-\partial_{x x}^{2}\right)(\lambda u)=(k+1) u^{k-1} u_{x} u_{x x}+u^{k} u_{x x x}, \quad x \in \mathbb{T}, t>0$.

For instance, the weakly dissipative $\mathrm{CH}$ equation, i.e. (1.4) for $k=1$, has been studied in $[49,65]$. For the Novikov equation (1.3) with the same weakly dissipative term $\left(1-\partial_{x x}^{2}\right)(\lambda u)$, we can also refer to [49].

In this work, we assume that the energy exchange mechanisms are connected with randomness to account for external stochastic influence. We are interested in the case where in the term $\left(1-\partial_{x x}^{2}\right)(\lambda u)$ the deterministic parameter $\lambda$ is substituted by a formal cylindrical Wiener process (cf. Sect. 2 for precise definitions), and where the previously linear dependency on $u$ is replaced by a non-autonomous and nonlinear term $h(t, u)$. Thus, we consider the Cauchy problem for (1.1) on the torus $\mathbb{T}$, with random initial data $u_{0}=u_{0}(\omega, x)$. Applying the operator $\left(1-\partial_{x x}^{2}\right)^{-1}$ to $(1.1)$, we reformulate the problem as the stochastic evolution 


$$
\left\{\begin{array}{c}
\mathrm{d} u+\left[u^{k} \partial_{x} u+F(u)\right] \mathrm{d} t=h(t, u) \mathrm{d} \mathcal{W}, \quad x \in \mathbb{T}, t>0, k \geq 1 \\
u(\omega, 0, x)=u_{0}(\omega, x), \quad x \in \mathbb{T}
\end{array}\right.
$$

with $F(u)=F_{1}(u)+F_{2}(u)+F_{3}(u)$ and

$$
\begin{aligned}
& F_{1}(u)=\left(1-\partial_{x x}^{2}\right)^{-1} \partial_{x}\left(u^{k+1}\right), \\
& F_{2}(u)=\frac{2 k-1}{2}\left(1-\partial_{x x}^{2}\right)^{-1} \partial_{x}\left(u^{k-1} u_{x}^{2}\right), \\
& F_{3}(u)=\frac{k-1}{2}\left(1-\partial_{x x}^{2}\right)^{-1}\left(u^{k-2} u_{x}^{3}\right) .
\end{aligned}
$$

Here we remark that the operator $\left(1-\partial_{x x}^{2}\right)^{-1}$ in $F(\cdot)$ is understood as

$$
\left[\left(1-\partial_{x x}^{2}\right)^{-1} f\right](x)=\left[G_{\mathbb{T}} * f\right](x) \text { for } f \in L^{2}(\mathbb{T}) \text { with } G_{\mathbb{T}}=\frac{\cosh \left(x-2 \pi\left[\frac{x}{2 \pi}\right]-\pi\right)}{2 \sinh (\pi)},
$$

where the convolution is on $\mathbb{T}$ only, and the symbol $[x]$ stands for the integer part of $x$.

The first objective of this paper is to analyze the local existence and uniqueness of pathwise solutions as well as blow-up criteria for problem (1.5) with nonlinear multiplicative noise (see Theorem 2.1). We note that for the $\mathrm{CH}$ equation with additive noise, existence and uniqueness has been obtained in [11]. For the stochastic modified $\mathrm{CH}$ equation with linear multiplicative noise, we refer to [10]. When the multiplicative noise is given by a one-dimensional Wiener process with $H^{s}$ diffusion coefficient, the stochastic $\mathrm{CH}$ equation was considered in second author's work [58]. In this paper, we consider the local existence and uniqueness of pathwise solutions to (1.5) as well as blow-up criteria for more general noise given by a cylindrical Wiener process with nonlinear coefficient.

The second major objective of the paper is to investigate whether stochastic perturbations can improve the dependence on initial data in the Cauchy problem (1.5). We notice that various instances of transport-type stochastic evolution laws have been studied with respect to the effect of noise on the regularity of their solutions. For example, we refer to $[21,23,53,54]$ for linear stochastic transport equations and to what concerns nonlinear stochastic conservation laws, we refer to $[27,50,51]$. In $[29,46,57,58]$ the dissipation of energy caused by linear multiplicative noise has been analyzed.

In contrast to these works we focus in this paper on the initial-data dependence in the Cauchy problem (1.5). Actually, much less is known on the noise effect with respect to the dependence on initial data. But the question whether (and how) noise can affect initial-data dependence is interesting. Formally speaking, regularization produced by noise may be seen like the deterministic regularization effect induced by adding a Laplacian. However, if one would add a real Laplacian to the governing equations, then by using techniques from the theory for semilinear parabolic equations, the dependence on initial data in some cases turns out to be more regular than being just continuous. For example, for the deterministic incompressible Euler equations, the dependence on initial data cannot be better than continuous [35], but for the deterministic incompressible Navier-Stokes equations with sufficiently large viscosity, it is at least Lipschitz continuous in sufficiently high Sobolev norms, see pp. 79-81 in [31]. This motivates us to study whether (and how) noise can affect initial-data dependence. Actually, to our knowledge, there are very few results in this direction. We can only refer to [59] for the stochastic Euler-Poincaré equation with respect to this problem. However, in this work, we have higher order nonlinearities, which requires more technical estimates. To 
analyze initial-data dependence, we introduce the concept of the stability of the exiting time (see Definition 2.2 below), cf. [59]. Then we show under some conditions on $h(t, u)$, that the multiplicative noise (in the Itô sense) cannot improve the stability of the exiting time, and, at the same time, improve the continuity of the map $u_{0} \mapsto u$ (see Theorem 2.2). It is worth noting that in deterministic cases, the issue of the optimal dependence of solutions (for example, the solution map is continuous but not uniformly continuous) to various nonlinear dispersive and integrable equations has been the subject of many papers. One of the first results of this type dates back at least as far as to Kato [42]. Indeed, Kato [42] proved that the solution map $u_{0} \mapsto u$ in $H^{s}(\mathbb{T})(s>3 / 2)$, given by the inviscid Burgers equation, is not Hölder continuous regardless of the Hölder exponent. Since then different techniques have been successfully applied to various nonlinear dispersive and integrable equations, see $[2,45,47]$ for example. Particularly, for the incompressible Euler equation, we refer to [35,60], and for $\mathrm{CH}$ type equations, we refer to $[33,34,61-63]$ and the references therein.

We conclude the paper with a discussion of time-global well-posedness for (1.5). For general noise terms, it is difficult to determine whether a local pathwise solution to (1.5) is globally defined. As shown in $[29,46,48,57,58]$, the linear noise $h(t, u) \mathrm{d} W=\beta u \mathrm{~d} W$, with $\beta \in \mathbb{R} \backslash\{0\}$ and $W$ being a standard 1-D Brownian motion, acts dissipatively for many SPDEs. Motivated by these works, we prove some results in Theorems 2.3 and 2.4 for the global dynamics of (1.5) with linear multiplicative noise, that is

$$
\left\{\begin{array}{l}
\mathrm{d} u+\left[u^{k} \partial_{x} u+F(u)\right] \mathrm{d} t=b(t) u \mathrm{~d} W, \quad x \in \mathbb{T}, t \in \mathbb{R}^{+}, k \geq 1, \\
u(\omega, 0, x)=u_{0}(\omega, x), \quad x \in \mathbb{T} .
\end{array}\right.
$$

\section{Preliminaries and Main Results}

\subsection{Noise with $H^{5}$ Coefficient}

We begin by introducing some notations. Throughout the paper, $(\Omega, \mathcal{F}, \mathbb{P})$ denotes a complete probability space, where $\mathbb{P}$ is the probability measure on $\Omega$ and $\mathcal{F}$ is a $\sigma$-algebra. Let $t>$ 0. $\sigma\left\{(x(\tau), y(\tau))_{\tau \in[0, t]}\right\}$ stands for the completion of the union $\sigma$-algebra generated by $(x(\tau), y(\tau))$. All stochastic integrals are defined in the Itô sense and $\mathbb{E} \cdot$ is the mathematical expectation of - with respect to $\mathbb{P}$. For some separable Banach space $X, \mathcal{B}(X)$ denotes the Borel sets of $X$, and $\operatorname{Pr}(X)$ stands for the collection of Borel probability measures on $X$. For $E \subseteq X, \mathbf{1}_{E}$ is the indicator function on $E$.

$L^{2}(\mathbb{T})$ is the usual square integrable function space on $\mathbb{T}$. For $s \in \mathbb{R}, D^{s}=\left(1-\partial_{x x}^{2}\right)^{s / 2}$ is defined by $\widehat{D^{s} f}(k)=\left(1+k^{2}\right)^{s / 2} \widehat{f}(k)$, where $\widehat{g}$ denote the Fourier transform of $g$ on $\mathbb{T}$. The Sobolev space $H^{s}(\mathbb{T})$ is defined as

$$
H^{s}(\mathbb{T}) \triangleq\left\{f \in L^{2}(\mathbb{T}):\|f\|_{H^{s}(\mathbb{T})}^{2}=\sum_{k \in \mathbb{Z}}\left(1+k^{2}\right)^{s}|\widehat{f}(k)|^{2}<+\infty\right\},
$$

and the inner product on $H^{s}(\mathbb{T})$ is $(f, g)_{H^{s}} \triangleq \sum_{k \in \mathbb{Z}}\left(1+k^{2}\right)^{s} \widehat{f}(k) \cdot \widehat{g}(k)=\left(D^{s} f, D^{s} g\right)_{L^{2}}$. For function spaces on $\mathbb{T}$, we will drop $\mathbb{T}$ if there is no ambiguity. We will use $\lesssim$ to denote estimates that hold up to some universal deterministic constant which may change from line to line but whose meaning is clear from the context. For linear operators $A$ and $B,[A, B]$ stands for the commutator of $A$ and $B$, i.e., $[A, B]=A B-B A$.

We briefly recall some aspects of the stochastic analysis theory which we use below. We refer the readers to $[19,25,40]$ for an extended treatment of this subject. 
We call $\mathcal{S}=\left(\Omega, \mathcal{F}, \mathbb{P},\left\{\mathcal{F}_{t}\right\}_{t \geq 0}, \mathcal{W}\right)$ a stochastic basis, where $\left\{\mathcal{F}_{t}\right\}_{t \geq 0}$ is a rightcontinuous filtration on $(\Omega, \mathcal{F})$ such that $\left\{\mathcal{F}_{0}\right\}$ contains all the $\mathbb{P}$-negligible subsets and $\mathcal{W}(t)=\mathcal{W}(\omega, t), \omega \in \Omega$ is a cylindrical Brownian motion, defined on an auxiliary Hilbert space $U$, which is adapted to $\left\{\mathcal{F}_{t}\right\}_{t \geq 0}$. Formally, if $\left\{e_{k}\right\}$ is a complete orthonormal basis of $U$ and $\left\{W_{k}\right\}_{k \geq 1}$ is a sequence of mutually independent standard one-dimensional Brownian motions, then one may define

$$
\mathcal{W}=\sum_{k=1}^{\infty} e_{k} W_{k} \mathbb{P}-\text { a.s. }
$$

To guarantee the convergence of the (formal) summation above, we consider a larger separable Hilbert space $U_{0}$ such that the canonical embedding $U \hookrightarrow U_{0}$ is Hilbert-Schmidt. Then we have that for any $T>0$, cf. [19,25,41],

$$
\mathcal{W}=\sum_{k=1}^{\infty} e_{k} W_{k} \in C\left([0, T] ; U_{0}\right) \mathbb{P}-a . s .
$$

To define the Itô stochastic integral

$$
\int_{0}^{\tau} G \mathrm{~d} \mathcal{W}=\sum_{k=1}^{\infty} \int_{0}^{\tau} G e_{k} \mathrm{~d} W_{k}
$$

on $H^{s}$, it is required (see e.g. [19,56]) for the predictable stochastic process $G$ to take values in the space of Hilbert-Schmidt operators from $U$ to $H^{s}$, denoted by $L_{2}\left(U ; H^{s}\right)$. For such $G,(2.1)$ is a well-defined continuous $H^{s}$-valued square integrable martingale such that for all almost surely bounded stopping times $\tau$ and for all $v \in H^{s}$,

$$
\left(\int_{0}^{\tau} G \mathrm{~d} \mathcal{W}, v\right)_{H^{s}}=\sum_{k=1}^{\infty} \int_{0}^{\tau}\left(G e_{k}, v\right)_{H^{s}} \mathrm{~d} W_{k} .
$$

Moreover, the desirable Burkholder-Davis-Gundy inequality in our case turns out to be

$$
\mathbb{E}\left(\sup _{t \in[0, T]}\left\|\int_{0}^{t} G \mathrm{~d} \mathcal{W}\right\|_{H^{s}}^{p}\right) \leq C(p, s) \mathbb{E}\left(\int_{0}^{T}\|G\|_{L_{2}\left(U ; H^{s}\right)}^{2} \mathrm{~d} t\right)^{\frac{p}{2}}, \quad p \geq 1 .
$$

or in terms of the coefficients,

$$
\mathbb{E}\left(\sup _{t \in[0, T]}\left\|\sum_{k=1}^{\infty} \int_{0}^{t} G e_{k} \mathrm{~d} W_{k}\right\|_{H^{s}}^{p}\right) \leq C(p, s) \mathbb{E}\left(\int_{0}^{T} \sum_{k=1}^{\infty}\left\|G e_{k}\right\|_{H^{s}}^{2} \mathrm{~d} t\right)^{\frac{p}{2}}, \quad p \geq 1 .
$$

Here we remark that the stochastic integral (2.1) does not depend on the choice of the space $U_{0}$, cf. $[19,56]$. For example, $U_{0}$ can be defined as

$$
U_{0}=\left\{v=\sum_{k=1}^{\infty} a_{k} e_{k}: \sum_{k=1}^{\infty} \frac{a_{k}^{2}}{k^{2}}<\infty\right\},\|v\|_{U_{0}}=\sum_{k=1}^{\infty} \frac{a_{k}^{2}}{k^{2}}
$$

\subsection{Definitions of the Solutions and Stability of the Exiting Times}

We now make the precise notion of a pathwise solution to (1.5). 
Definition 2.1 (Pathwise solutions) Let $\mathcal{S}=\left(\Omega, \mathcal{F}, \mathbb{P},\left\{\mathcal{F}_{t}\right\}_{t \geq 0}, \mathcal{W}\right)$ be a fixed stochastic basis. Let $s>3 / 2$ and $u_{0}$ be an $H^{s}$-valued $\mathcal{F}_{0}$-measurable random variable (relative to $\mathcal{S}$ ).

1. A local pathwise solution to (1.5) is a pair $(u, \tau)$, where $\tau \geq 0$ is a stopping time satisfying $\mathbb{P}\{\tau>0\}=1$ and $u: \Omega \times[0, \infty] \rightarrow H^{s}$ is an $\mathcal{F}_{t}$-predictable $H^{s}$-valued process satisfying

$$
u(\cdot \wedge \tau) \in C\left([0, \infty) ; H^{s}\right) \mathbb{P}-\text { a.s. },
$$

and for all $t>0$,

$$
u(t \wedge \tau)-u(0)+\int_{0}^{t \wedge \tau}\left[u^{k} \partial_{x} u+F(u)\right] \mathrm{d} t^{\prime}=\int_{0}^{t \wedge \tau} h\left(t^{\prime}, u\right) \mathrm{d} \mathcal{W} \mathbb{P}-\text { a.s. }
$$

2. The local pathwise solutions are said to be pathwise unique, if given any two pairs of local pathwise solutions $\left(u_{1}, \tau_{1}\right)$ and $\left(u_{2}, \tau_{2}\right)$ with $\mathbb{P}\left\{u_{1}(0)=u_{2}(0)\right\}=1$, we have

$$
\mathbb{P}\left\{u_{1}(t, x)=u_{2}(t, x), \forall(t, x) \in\left[0, \tau_{1} \wedge \tau_{2}\right] \times \mathbb{T}\right\}=1 .
$$

3. Additionally, $\left(u, \tau^{*}\right)$ is called a maximal pathwise solution to (1.5) if $\tau^{*}>0$ almost surely and if there is an increasing sequence $\tau_{n} \rightarrow \tau^{*}$ such that for any $n \in \mathbb{N},\left(u, \tau_{n}\right)$ is a pathwise solution to (1.5) and on the set $\left\{\tau^{*}<\infty\right\}$,

$$
\sup _{t \in\left[0, \tau_{n}\right]}\|u\|_{H^{s}} \geq n
$$

4. If $\left(u, \tau^{*}\right)$ is a maximal pathwise solution and $\tau^{*}=\infty$ almost surely, then we say that the pathwise solution exists globally.

A major result of this paper is a (negative) dependence statement for the initial data of the solutions. Precisely, it refers to the stability of a point in time when the solution leaves a certain range. This point in time is called exiting time and we introduce

Definition 2.2 (Stability of exiting time) Let $s>3 / 2$ and $\mathcal{S}=\left(\Omega, \mathcal{F}, \mathbb{P},\left\{\mathcal{F}_{t}\right\}_{t \geq 0}, \mathcal{W}\right)$ be a fixed stochastic basis. Let $u_{0}$ be an $H^{s}$-valued $\mathcal{F}_{0}$-measurable random variable such that $\mathbb{E}\left\|u_{0}\right\|_{H^{s}}^{2}<\infty$. Assume that $\left\{u_{0, n}\right\}$ is any sequence of $H^{s}$-valued $\mathcal{F}_{0}$-measurable random variables satisfying $\mathbb{E}\left\|u_{0, n}\right\|_{H^{s}}^{2}<\infty$. For each $n$, let $u$ and $u_{n}$ be the unique pathwise solutions to (1.5) with initial value $u_{0}$ and $u_{0, n}$, respectively.

For any $R>0$ and $n \in \mathbb{N}$, we define the $R$-exiting times

$$
\tau_{n}^{R}:=\inf \left\{t \geq 0:\left\|u_{n}(t)\right\|_{H^{s}}>R\right\} \text { and } \tau^{R}:=\inf \left\{t \geq 0:\|u(t)\|_{H^{s}}>R\right\},
$$

where inf $\varnothing=\infty$. Furthermore,

1. If $u_{0, n} \rightarrow u_{0}$ in $H^{s} \mathbb{P}-a . s$. implies that

$$
\lim _{n \rightarrow \infty} \tau_{n}^{R}=\tau^{R} \mathbb{P}-\text { a.s. }
$$

then the $R$-exiting time of $u$ is said to be stable.

2. If $u_{0, n} \rightarrow u_{0}$ in $H^{s^{\prime}}$ for all $s^{\prime}<s$ almost surely implies that (2.2) holds true, the $R$-exiting time of $u$ is said to be strongly stable.

\subsection{Assumptions}

For our results on existence of pathwise solutions, on the stability of exiting times and global well-posedness of (1.1), we rely in the following sections on generic but slightly different 
assumptions concerning the data in (1.1). These are summarized here, for a comment on possible relaxed versions we refer to Remark 3.1.

Assumption $\mathbf{A}_{1}$. We assume that $h:[0, \infty) \times H^{s} \ni(t, u) \mapsto h(t, u) \in L_{2}\left(U ; H^{s}\right)$ for $u \in H^{s}$ with $s \geq 0$ such that if $u: \Omega \times[0, T] \rightarrow H^{s}$ is predictable, then $h(t, u)$ is also predictable. Furthermore, we assume the following:

1. There is an increasing and locally bounded function $f(\cdot):[0,+\infty) \rightarrow[0,+\infty)$ with $f(0)=0$ such that for any $t>0$ and $u \in H^{s}$ with $s>\frac{1}{2}$,

$$
\|h(t, u)\|_{L_{2}\left(U ; H^{s}\right)} \leq f\left(\|u\|_{W^{1, \infty}}\right)\left(1+\|u\|_{H^{s}}\right) .
$$

Particularly, if $h$ does not depend on $u$, i.e., the additive noise case, then the condition $f(0)=0$ can be removed.

2. There is an increasing locally bounded function $g(\cdot):[0,+\infty) \rightarrow[0,+\infty)$, such that for any $t>0$ and $u \in H^{s}$ with $s>\frac{1}{2}$,

$$
\|h(t, u)-h(t, v)\|_{L_{2}\left(U ; H^{s}\right)} \leq g\left(\|u\|_{H^{s}}+\|v\|_{H^{s}}\right)\|u-v\|_{H^{s}} .
$$

Assumption $\mathbf{A}_{2}$. When we consider the initial-data dependence problem for (1.5) in Sect. 4, we need a similar assumption on $h(t, \cdot)$. For $s \geq 0$ and $u \in H^{s}, h:[0, \infty) \times H^{s} \ni(t, u) \mapsto$ $h(t, u) \in L_{2}\left(U ; H^{s}\right)$ for $u \in H^{s}$ with $s \geq 0$ such that if $u: \Omega \times[0, T] \rightarrow H^{s}$ is predictable, then $h(t, u)$ is also predictable. Moreover, for all $t \geq 0$, we assume

$$
\|h(t, u)\|_{L_{2}\left(U ; H^{s}\right)} \leq\|F(u)\|_{H^{s}}, \quad\|h(t, u)-h(t, v)\|_{L_{2}\left(U ; H^{s}\right)} \leq\|F(u)-F(v)\|_{H^{s}},
$$

where $F(\cdot)$ is defined by (1.6).

Assumption $\mathbf{A}_{3}$. When considering (1.8) with non-autonomous linear noise $b(t) u \mathrm{~d} W$, we assume that $b(t) \in C([0, \infty))$ such that $0<b_{*} \leq b^{2}(0) \leq \sup _{t \geq 0} b^{2}(t) \leq b^{*}$ for some $b_{*}, b^{*} \in \mathbb{R}$.

\subsection{Main Results}

In this section we summarize our major contributions providing proofs later in the remainder of the paper.

Theorem 2.1 Let $s>3 / 2, k \geq 1$ and let $h(t, u)$ satisfy Assumption $A_{1}$. For a given stochastic basis $\mathcal{S}=\left(\Omega, \mathcal{F}, \mathbb{P},\left\{\mathcal{F}_{t}\right\}_{t \geq 0}, \mathcal{W}\right)$, if $u_{0}$ is an $H^{s}$-valued $\mathcal{F}_{0}$-measurable random variable satisfying $\mathbb{E}\left\|u_{0}\right\|_{H^{s}}^{2}<\infty$, then there is a local unique pathwise solution $(u, \tau)$ to $(1.5)$ in the sense of Definition 2.1 with

$$
u(\cdot \wedge \tau) \in L^{2}\left(\Omega ; C\left([0, \infty) ; H^{s}\right)\right) .
$$

Moreover, $(u, \tau)$ can be extended to a unique maximal pathwise solution $\left(u, \tau^{*}\right)$ with

$$
\mathbf{1}_{\left\{\tau^{*}<\infty\right\}}=\mathbf{1}_{\left\{\lim \sup _{t \rightarrow \tau^{*}}\|u(t)\|_{W^{1, \infty}}=\infty\right\}} \mathbb{P}-\text { a.s. }
$$

Remark 2.1 We remark here that $F_{3}(u)$ in (1.6) will disappear when $k=1$. The proof for Theorem 2.1 combines the techniques as employed in the papers [3-5,18,20,29,58]. However, the Faedo-Galerkin method used e.g. in [20,29] cannot be utilized directly since in (1.5), we do not have additional constraints like incompressibility, which guarantees the global existence of an approximate solution (see, e.g. [22,29]). Without this, we need to find a positive lower bound for the lifespan of the approximate solutions, which is generally not clear. Particularly, 
for our case, this difficulty will be overcome by constructing a suitable approximation scheme and establishing an appropriate blow-up criterium, which applies not only for $u$, but also for the approximate solution $u_{\varepsilon}$. This idea is transferred from the recent work [18] on deriving blow-up criteria.

The next result addresses the dependence of the solution on initial data giving at least a partial answer.

Theorem 2.2 (Weak instability) Consider the periodic initial value problem (1.5), where $F(\cdot)$ is given by (1.6) with $k \geq 1$. Let $\mathcal{S}=\left(\Omega, \mathcal{F}, \mathbb{P},\left\{\mathcal{F}_{t}\right\}_{t \geq 0}, \mathcal{W}\right)$ be a fixed stochastic basis and let $s>3 / 2$. If $h$ satisfies Assumption $A_{2}$, then at least one of the following properties holds true.

1. For any $R \gg 1$, the R-exiting time is not strongly stable for the zero solution in the sense of Definition 2.2;

2. The solution map $u_{0} \mapsto u$ defined by solving (1.5) is not uniformly continuous as a map from $L^{2}\left(\Omega, H^{s}\right)$ into $L^{2}\left(\Omega ; C\left([0, T] ; H^{s}\right)\right)$ for any $T>0$. More precisely, there exist two sequences of solutions $u_{1, n}(t)$ and $u_{2, n}(t)$, and two sequences of stopping times $\tau_{1, n}$ and $\tau_{2, n}$, such that

(a) $\mathbb{P}\left\{\tau_{i, n}>0\right\}=1$ for each $n>1$ and $i=1,2$. Besides,

$$
\lim _{n \rightarrow \infty} \tau_{1, n}=\lim _{n \rightarrow \infty} \tau_{2, n}=\infty \mathbb{P}-\text { a.s. }
$$

(b) For $i=1,2, u_{i, n} \in C\left(\left[0, \tau_{i, n}\right] ; H^{s}\right) \mathbb{P}-$ a.s., and

$$
\mathbb{E}\left(\sup _{t \in\left[0, \tau_{1, n}\right]}\left\|u_{1, n}(t)\right\|_{H^{s}}^{2}+\sup _{t \in\left[0, \tau_{2, n}\right]}\left\|u_{2, n}(t)\right\|_{H^{s}}^{2}\right) \lesssim 1 .
$$

(c) At $t=0$,

$$
\lim _{n \rightarrow \infty} \mathbb{E}\left\|u_{1, n}(0)-u_{2, n}(0)\right\|_{H^{s}}^{2}=0
$$

(d) For any $T>0$,

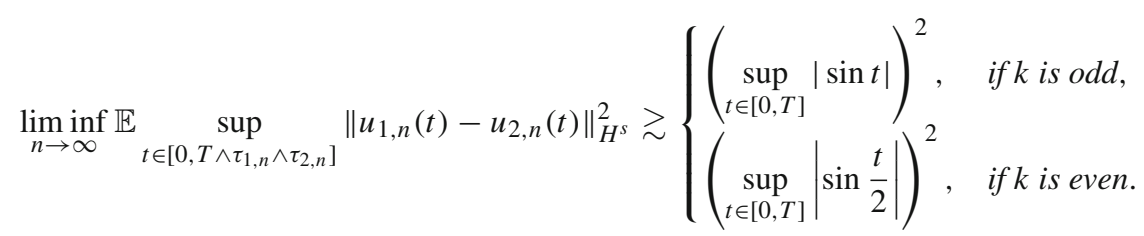

Remark 2.2 To prove Theorem 2.2, we assume that for some $R_{0} \gg 1$, the $R_{0}$-exiting time is strongly stable at the zero solution. Then we will construct an example to show that the solution map $u_{0} \mapsto u$ defined by (1.5) is not uniformly continuous. This example involves the construction (for each $s>3 / 2$ ) of two sequences of solutions which are converging at time zero but remain far apart at any later time. Therefore we will first construct two sequences of approximation solutions $u^{i, n}(i \in\{1,2\})$ such that the actual solutions $u_{i, n}$ starting from $u_{i, n}(0)=u^{i, n}(0)$ satisfy that as $n \rightarrow \infty$,

$$
\lim _{n \rightarrow \infty} \mathbb{E} \sup _{\left[0, \tau_{i, n}\right]}\left\|u_{i, n}-u^{i, n}\right\|_{H^{s}}=0,
$$


where $u_{i, n}$ exists at least on $\left[0, \tau_{i, n}\right]$. If we obtain (2.12), then we can estimate the approximation solutions instead of the actual solutions and obtain (2.11). In deterministic case, for other works using the method of approximate solutions for studying dependence on initial data, we refer the reader to $[2,34,47,61,63]$ and the references therein. However, in contrast to deterministic cases where lifespan estimate can be achieved (see (4.7)-(4.8) in [62] and (3.8)-(3.9) in [63] for example), it is not clear $\inf _{n} \tau_{i, n}>0$ almost surely in stochastic setting. Therefore we are motivated to introduce the definition on the stability of the exiting time (see Definition 2.2). Then we find that the property $\inf _{n} \tau_{i, n}>0$ can be connected with the stability property of the exiting time of the zero solution. Then we estimate the error in $H^{2 s-\sigma}$ and $H^{\sigma}$ with suitable $\sigma$. Different from [59], the problem (1.5) has nonlinearities of order $k+1$ and (2.12) should depend on $k$. Therefore more technical estimates are needed (see Lemma 4.1 and (4.17) for example). Finally we use interpolation to derive (2.12). Theorem 2.2 shows that one cannot expect too much for the issue of the dependence on initial data. More precisely, we cannot expect to improve the stability of the exiting time for the zero solution, and simultaneously to improve the continuous dependence of solutions on initial data.

Finally, we focus on (1.8). For the issue of global existence, we have

Theorem 2.3 (Global existence: Case 1) Let $k \geq 1$ and $s>3 / 2$. Let $b(t)$ satisfy Assumption $A_{3}$ and $\mathcal{S}=\left(\Omega, \mathcal{F}, \mathbb{P},\left\{\mathcal{F}_{t}\right\}_{t \geq 0}, W\right)$ be a fixed stochastic basis. Assume $u_{0}$ to be an $H^{s}$-valued $\mathcal{F}_{0}$-measurable random variable satisfying $\mathbb{E}\left\|u_{0}\right\|_{H^{s}}^{2}<\infty$. Let $K$ be a constant such that $\|\cdot\|_{W^{1, \infty}}<K\|\cdot\|_{H^{s}}$. Then there is a $C=C(s)>1$ such that for any $R>1$ and $\lambda_{1}>2$, if $\left\|u_{0}\right\|_{H^{s}}<\frac{1}{R K}\left(\frac{b_{*}}{C \lambda_{1}}\right)^{1 / k}$ almost surely, then (1.8) has a maximal pathwise solution $\left(u, \tau^{*}\right)$ satisfying for any $\lambda_{2}>\frac{2 \lambda_{1}}{\lambda_{1}-2}$ the estimate

$$
\mathbb{P}\left\{\|u(t)\|_{H^{s}}<\frac{1}{K}\left(\frac{b_{*}}{C \lambda_{1}}\right)^{1 / k} \mathrm{e}^{-\frac{\left(\left(\lambda_{1}-2\right) \lambda_{2}-2 \lambda_{1}\right)}{2 \lambda_{1} \lambda 2} \int_{0}^{t} b^{2}\left(t^{\prime}\right) \mathrm{d} t^{\prime}} \text { for all } t>0\right\} \geq 1-\left(\frac{1}{R}\right)^{2 / \lambda_{2}} .
$$

Remark 2.3 Here we notice that if $k=1$, then $F_{3}(u)$ in (1.6) will disappear. Motivated by the recent papers [29,57,58], where the linear noise $\beta u \mathrm{~d} W$ with $\beta \in \mathbb{R} \backslash\{0\}$ is considered, we focus on the non-autonomous linear multiplicative noise case, namely (1.8). We transform (1.8) to a non-autonomous random system (5.2). Although the stochastic integral is absent in (5.2), to extend the deterministic results to the stochastic setting, we need to overcome a few technical difficulties since the system is not only random but also non-autonomous. In this work, we manage to gain some estimates and asymptotic limits of the Girsanov type processes (see e.g., (5.6), (5.8), (5.10) and Lemma A.7), which enable us to apply the energy estimate pathwisely (namely for a.e. $\omega \in \Omega$ ) and obtain Theorem 2.3.

Theorem 2.4 (Global existence: Case 2) Let $\mathcal{S}=\left(\Omega, \mathcal{F}, \mathbb{P},\left\{\mathcal{F}_{t}\right\}_{t \geq 0}, W\right)$ be a fixed stochastic basis. Let $s>3$ and $b(t)$ satisfy Assumption $A_{3}$. Assume $u_{0}$ to be an $H^{s}$-valued, $\mathcal{F}_{0^{-}}$ measurable random variable satisfying $\mathbb{E}\left\|u_{0}\right\|_{H^{s}}^{2}<\infty$. If $u_{0}$ satisfies

$$
\mathbb{P}\left\{\left(1-\partial_{x x}^{2}\right) u_{0}(x)>0, \forall x \in \mathbb{T}\right\}=p, \mathbb{P}\left\{\left(1-\partial_{x x}^{2}\right) u_{0}(x)<0, \forall x \in \mathbb{T}\right\}=q,
$$

for some $p, q \in[0,1]$, then the corresponding maximal pathwise solution $\left(u, \tau^{*}\right)$ to $(1.8)$ satisfies

$$
\mathbb{P}\left\{\tau^{*}=\infty\right\} \geq p+q .
$$

That is to say, $\mathbb{P}\{u$ exists globally $\} \geq p+q$. 


\section{Proof for Theorem 2.1}

\subsection{Blow-Up Criteria}

Let us postpone the proof for existence and uniqueness of solutions to (1.5) to Sect. 3.2. We will first prove the blow-up criteria, since some estimates will be used later. Motivated by [18], we first consider the relationship between the blow-up time of $\|u(t)\|_{H^{s}}$ and the blow-up time of $\|u(t)\|_{W^{1, \infty}}$ for (1.5). Even though one might expect that the $\|u(t)\|_{H_{s}}$ norm may blow up earlier than $\|u(t)\|_{W^{1, \infty}}$, the following result shows that this is not true.

Lemma 3.1 Let $\left(u, \tau^{*}\right)$ be the unique maximal pathwise solution to (1.5). Then the realvalued stochastic process $\|u\|_{W^{1, \infty}}$ is also $\mathcal{F}_{t}$-adapted. Besides, for any $m, n \in \mathbb{Z}^{+}$, define

$$
\tau_{1, m}=\inf \left\{t \geq 0:\|u(t)\|_{H^{s}} \geq m\right\}, \quad \tau_{2, n}=\inf \left\{t \geq 0:\|u(t)\|_{W^{1, \infty}} \geq n\right\} .
$$

Moreover, let $\tau_{1}=\lim _{m \rightarrow \infty} \tau_{1, m}$ and $\tau_{2}=\lim _{n \rightarrow \infty} \tau_{2, n}$, then we have

$$
\tau_{1}=\tau_{2} \mathbb{P}-\text { a.s. }
$$

As a corollary, $\boldsymbol{1}_{\left\{\lim _{t \rightarrow \tau^{*}}\|u(t)\|_{W^{1}, \infty}=\infty\right\}}=\mathbf{1}_{\left\{\tau^{*}<\infty\right\}} \mathbb{P}-$ a.s.

Proof To begin with, we see that $u(\cdot \wedge \tau) \in C\left([0, \infty) ; H^{s}\right)$ means that for any $t \in[0, \tau]$,

$$
[u(t)]^{-1}(Y)=[u(t)]^{-1}\left(H^{s} \cap Y\right), \forall Y \in \mathcal{B}\left(W^{1, \infty}\right) .
$$

Therefore $u(t)$, as a $W^{1, \infty}$-valued process, is also $\mathcal{F}_{t}$-adapted. We then infer from the embed$\operatorname{ding} H^{s} \hookrightarrow W^{1, \infty}$ for $s>3 / 2$ that for some $M>0$ and $m \in \mathbb{N}$,

$$
\sup _{t \in\left[0, \tau_{1, m}\right]}\|u(t)\|_{W^{1, \infty}} \leq M \sup _{t \in\left[0, \tau_{1, m}\right]}\|u(t)\|_{H^{s}} \leq([M]+1) m,
$$

where $[M]$ means the integer part of $M$. Therefore we have $\tau_{1, m} \leq \tau_{2,([M]+1) m} \leq \tau_{2} \mathbb{P}-$ a.s., which means that $\tau_{1} \leq \tau_{2} \mathbb{P}-$ a.s. Now we only need to prove $\tau_{2} \leq \tau_{1} \mathbb{P}-$ a.s. It is easy to see that for all $n_{1}, n_{2} \in \mathbb{Z}^{+}$,

$$
\begin{aligned}
& \left\{\sup _{t \in\left[0, \tau_{2, n_{1}} \wedge n_{2}\right]}\|u(t)\|_{H^{s}}<\infty\right\}=\bigcup_{m \in \mathbb{Z}^{+}}\left\{\sup _{t \in\left[0, \tau_{2, n_{1}} \wedge n_{2}\right]}\|u(t)\|_{H^{s}}<m\right\} \\
& \subset \bigcup_{m \in \mathbb{Z}^{+}}\left\{\tau_{2, n_{1}} \wedge n_{2} \leq \tau_{1, m}\right\} .
\end{aligned}
$$

Since

$$
\bigcup_{m \in \mathbb{Z}^{+}}\left\{\tau_{2, n_{1}} \wedge n_{2} \leq \tau_{1, m}\right\} \subset\left\{\tau_{2, n_{1}} \wedge n_{2} \leq \tau_{1}\right\}
$$

we see that if

$$
\mathbb{P}\left\{\sup _{t \in\left[0, \tau_{2, n_{1}} \wedge n_{2}\right]}\|u(t)\|_{H^{s}}<\infty\right\}=1, \quad \forall n_{1}, n_{2} \in \mathbb{Z}^{+}
$$

holds true, then for all $n_{1}, n_{2} \in \mathbb{Z}^{+}, \mathbb{P}\left\{\tau_{2, n_{1}} \wedge n_{2} \leq \tau_{1}\right\}=1$ and

$$
\mathbb{P}\left\{\tau_{2} \leq \tau_{1}\right\}=\mathbb{P}\left\{\bigcap_{n_{1} \in \mathbb{Z}^{+}}\left\{\tau_{2, n_{1}} \leq \tau_{1}\right\}\right\}=\mathbb{P}\left\{\bigcap_{n_{1}, n_{2} \in \mathbb{Z}^{+}}\left\{\tau_{2, n_{1}} \wedge n_{2} \leq \tau_{1}\right\}\right\}=1 .
$$


Since (3.2) requires the assumption (3.1), it suffices to prove (3.1). However, we can not directly apply the Itô formula for $\|u\|_{H^{s}}^{2}$ to get control of $\mathbb{E}\|u(t)\|_{H^{s}}^{2}$ since we only have $u \in H^{s}$ and $u^{k} u_{x} \in H^{s-1}$. Therefore the well known Itô formula in general Hilbert space can not be used directly, see [19, Theorem 4.32] or [25, Theorem 2.10] for example. We will use the mollifier operator $T_{\varepsilon}$ defined in Appendix A to overcome this obstacle. Indeed, applying $T_{\varepsilon}$ to (1.5) and using the Itô formula for $\left\|T_{\varepsilon} u\right\|_{H^{s}}^{2}$, we have that for any $t>0$,

$$
\begin{aligned}
\mathrm{d}\left\|T_{\varepsilon} u(t)\right\|_{H^{s}}^{2}= & \left(T_{\varepsilon} h(t, u) \mathrm{d} \mathcal{W}, T_{\varepsilon} u\right)_{H^{s}}-2\left(D^{s} T_{\varepsilon}\left[u^{k} u\right], D^{s} T_{\varepsilon} u\right)_{L^{2}} \mathrm{~d} t \\
& -2\left(D^{s} T_{\varepsilon} F(u), D^{s} T_{\varepsilon} u\right)_{L^{2}} \mathrm{~d} t+\left\|T_{\varepsilon} h(t, u)\right\|_{L_{2}\left(U ; H^{s}\right)}^{2} \mathrm{~d} t .
\end{aligned}
$$

Therefore for any $n_{1}, n_{2} \geq 1$ and $t \in\left[0, \tau_{2, n_{1}} \wedge n_{2}\right]$,

$$
\begin{aligned}
\left\|T_{\varepsilon} u(t)\right\|_{H^{s}}^{2}-\left\|T_{\varepsilon} u(0)\right\|_{H^{s}}^{2}= & 2 \sum_{j=1}^{\infty} \int_{0}^{t}\left(D^{s} T_{\varepsilon} h(t, u) e_{j}, D^{s} T_{\varepsilon} u\right)_{L^{2}} \mathrm{~d} W_{j} \\
& -2 \int_{0}^{t}\left(D^{s} T_{\varepsilon}\left[u^{k} \partial_{x} u\right], D^{s} T_{\varepsilon} u\right)_{L^{2}} \mathrm{~d} t \\
& -2 \int_{0}^{t}\left(D^{s} T_{\varepsilon} F(u), D^{s} T_{\varepsilon} u\right)_{L^{2}} \mathrm{~d} t \\
& +\int_{0}^{t} \sum_{k=1}^{\infty}\left\|D^{s} T_{\varepsilon} h(t, u) e_{j}\right\|_{L^{2}}^{2} \mathrm{~d} t \\
= & \int_{0}^{t} \sum_{j=1}^{\infty} L_{1, j} \mathrm{~d} W_{j}+\sum_{i=2}^{4} \int_{0}^{t} L_{i} \mathrm{~d} t,
\end{aligned}
$$

where $\left\{e_{k}\right\}$ is the complete orthonormal basis of $U$. On account of the Burkholder-DavisGundy inequality, we arrive at

$$
\begin{aligned}
& \mathbb{E} \sup _{t \in\left[0, \tau_{2, n_{1}} \wedge n_{2}\right]}\left\|T_{\varepsilon} u(t)\right\|_{H^{s}}^{2} \leq \mathbb{E}\left\|T_{\varepsilon} u_{0}\right\|_{H^{s}}^{2}+C \mathbb{E}\left(\int_{0}^{\tau_{2, n_{1}} \wedge n_{2}}\left|\sum_{j=1}^{\infty} L_{1, j}\right|^{2} \mathrm{~d} t\right)^{\frac{1}{2}} \\
& \quad+\sum_{i=2}^{4} \mathbb{E} \int_{0}^{\tau_{2, n_{1}} \wedge n_{2}}\left|L_{i}\right| \mathrm{d} t .
\end{aligned}
$$

Then (2.3), (A.3) and the stochastic Fubini theorem [19] lead to

$$
\mathbb{E}\left(\int_{0}^{\tau_{2, n_{1}} \wedge n_{2}}\left|\sum_{j=1}^{\infty} L_{1, j}\right|^{2} \mathrm{~d} t\right)^{\frac{1}{2}} \leq \frac{1}{2} \mathbb{E} \sup _{t \in\left[0, \tau_{2, n_{1}} \wedge n_{2}\right]}\left\|T_{\varepsilon} u\right\|_{H^{s}}^{2}+C f^{2}\left(n_{1}\right) \int_{0}^{n_{2}}\left(1+\mathbb{E}\|u\|_{H^{s}}^{2}\right) \mathrm{d} t .
$$

For $L_{2}$, we notice that $T_{\varepsilon}$ satisfies (A.1), (A.2) and (A.3). Then it follows from Lemmas A.1, A.2 and A.3, integration by parts and $H^{s} \hookrightarrow W^{1, \infty}$ that

$$
\begin{aligned}
& \left(D^{s} T_{\varepsilon}\left[u^{k} u_{x}\right], D^{s} T_{\varepsilon} u\right)_{L^{2}} \\
= & \left(\left[D^{s}, u^{k}\right] u_{x}, D^{s} T_{\varepsilon}^{2} u\right)_{L^{2}}+\left(\left[T_{\varepsilon}, u^{k}\right] D^{s} u_{x}, D^{s} T_{\varepsilon} u\right)_{L^{2}}+\left(u^{k} D^{s} T_{\varepsilon} u_{x}, D^{s} T_{\varepsilon} u\right)_{L^{2}} \\
\leq & C\|u\|_{W^{1, \infty}}^{k}\|u\|_{H^{s}}^{2},
\end{aligned}
$$


which implies

$$
\mathbb{E} \int_{0}^{\tau_{2, n_{1}} \wedge n_{2}}\left|L_{2}\right| \mathrm{d} t \leq C n_{1}^{k} \int_{0}^{n_{2}}\left(1+\mathbb{E}\|u\|_{H^{s}}^{2}\right) \mathrm{d} t
$$

Similarly, it follows from Lemma A.5 and the assumption (2.3) that

$$
\mathbb{E} \int_{0}^{\tau_{2, n_{1}} \wedge n_{2}}\left|L_{3}\right|+\left|L_{4}\right| \mathrm{d} t \leq C\left(n_{1}^{k}+f^{2}\left(n_{1}\right)\right) \int_{0}^{n_{2}}\left(1+\mathbb{E}\|u\|_{H^{s}}^{2}\right) \mathrm{d} t .
$$

Therefore we combine the above estimates with using (A.3) to have

$$
\mathbb{E} \sup _{t \in\left[0, \tau_{2, n_{1}} \wedge n_{2}\right]}\left\|T_{\varepsilon} u(t)\right\|_{H^{s}}^{2} \leq C \mathbb{E}\left\|u_{0}\right\|_{H^{s}}^{2}+C \int_{0}^{n_{2}}\left(1+\mathbb{E} \sup _{t^{\prime} \in\left[0, t \wedge \tau_{2, n_{1}}\right]}\left\|u\left(t^{\prime}\right)\right\|_{H^{s}}^{2}\right) \mathrm{d} t,
$$

where $C=C\left(n_{1}, k\right)$ through $n_{1}^{k}$ and $n_{1}^{k}+f^{2}\left(n_{1}\right)$. Since the right hand side of the above estimate does not depend on $\varepsilon$, and $T_{\varepsilon} u$ tends to $u$ in $C\left([0, T], H^{s}\right)$ for any $T>0$ almost surely as $\varepsilon \rightarrow 0$, one can send $\varepsilon \rightarrow 0$ to obtain

$$
\mathbb{E} \sup _{t \in\left[0, \tau_{2, n_{1}} \wedge n_{2}\right]}\|u(t)\|_{H^{s}}^{2} \leq C \mathbb{E}\left\|u_{0}\right\|_{H^{s}}^{2}+C \int_{0}^{n_{2}}\left(1+\mathbb{E} \sup _{t^{\prime} \in\left[0, t \wedge \tau_{2, n_{1}}\right]}\left\|u\left(t^{\prime}\right)\right\|_{H^{s}}^{2}\right) \mathrm{d} t .
$$

Then Gronwall's inequality shows that for each $n_{1}, n_{2} \in \mathbb{Z}^{+}$, there is a constant $C=$ $C\left(n_{1}, n_{2}, u_{0}, k\right)>0$ such that

$$
\mathbb{E} \sup _{t \in\left[0, \tau_{2, n_{1}} \wedge n_{2}\right]}\|u(t)\|_{H^{s}}^{2}<C\left(n_{1}, n_{2}, u_{0}, k\right),
$$

which gives (3.1). Now we prove (2.7). Let us assume the existence and uniqueness first. We notice that for fixed $m, n>0$, even if $\mathbb{P}\left\{\tau_{1, m}=0\right\}$ or $\mathbb{P}\left\{\tau_{2, n}=0\right\}$ is larger than 0 , for a.e. $\omega \in \Omega$, there is $m>0$ or $n>0$ such that $\tau_{1, m}, \tau_{2, n}>0$. By continuity of $\|u(t)\|_{H^{s}}$ and the uniqueness of $u$, it is easy to check that $\tau_{1}=\tau_{2}$ is actually the maximal existence time $\tau^{*}$ of $u$ in the sense of Definition 2.1. Consequently, we obtain the desired blow-up criteria.

\subsection{Sketch of the Proof for Theorem 2.1}

Since the main idea of proving Theorem 2.1 follows standard ideas and it is very similar to $[58,59]$, here we only give a sketch of the main steps.

1. (Approximate solutions) The first step is to construct a suitable approximation scheme. For any $R>1$, we let $\chi_{R}(x):[0, \infty) \rightarrow[0,1]$ be a $C^{\infty}$ function such that $\chi_{R}(x)=1$ for $x \in[0, R]$ and $\chi_{R}(x)=0$ for $x>2 R$. Then we consider the following cut-off problem on $\mathbb{T}$,

$$
\left\{\begin{array}{l}
\mathrm{d} u+\chi_{R}\left(\|u\|_{W^{1, \infty}}\right)\left[u^{k} \partial_{x} u+F(u)\right] \mathrm{d} t=\chi_{R}\left(\|u\|_{W^{1, \infty}}\right) h(t, u) \mathrm{d} \mathcal{W} \\
u(\omega, 0, x)=u_{0}(\omega, x) \in H^{s}
\end{array}\right.
$$

From Lemma A.5, we see that the nonlinear term $F(u)$ preserves the $H^{s}$-regularity of $u \in H^{s}$ for any $s>3 / 2$. However, to apply the theory of SDEs in Hilbert space to (3.4), we will have to mollify the transport term $u^{k} \partial_{x} u$ since the product $u^{k} \partial_{x} u$ loses one regularity. To this end, we consider the following approximation scheme: 


$$
\left\{\begin{aligned}
\mathrm{d} u+G_{1, \varepsilon}(u) \mathrm{d} t & =G_{2}(u) \mathrm{d} \mathcal{W}, \quad x \in \mathbb{T}, t>0, \\
G_{1, \varepsilon}(u) & =\chi_{R}\left(\|u\|_{W^{1, \infty}}\right)\left[J_{\varepsilon}\left(\left(J_{\varepsilon} u\right)^{k} \partial_{x} J_{\varepsilon} u\right)+F(u)\right], \\
G_{2}(u) & =\chi_{R}\left(\|u\|_{W^{1, \infty}}\right) h(t, u), \\
u(0, x) & =u_{0}(x) \in H^{s}(\mathbb{T}),
\end{aligned}\right.
$$

where $J_{\varepsilon}$ is the Friedrichs mollifier defined in Appendix A. After mollifying the transport term $u^{k} u_{x}$, for a fixed stochastic basis $\mathcal{S}=\left(\Omega, \mathcal{F}, \mathbb{P},\left\{\mathcal{F}_{t}\right\}_{t \geq 0}, W\right)$ and for $u_{0} \in$ $L^{2}\left(\Omega ; H^{s}\right)$ with $s>3$, according to the existence theory of SDE in Hilbert space (see for example [56, Theorem 4.2.4 with Example 4.1.3] and [40]), (3.5) admits a unique solution $u_{\varepsilon} \in C\left(\left[0, T_{\varepsilon}\right), H^{s}\right) \mathbb{P}-$ a.s. Moreover, the uniform $L^{\infty}\left(\Omega ; W^{1, \infty}\right)$ condition provided by the cut-off function $\chi_{R}$ enables us to split the expectation $\mathbb{E}\left(\left\|u_{\varepsilon}\right\|_{H^{s}}^{2}\left\|u_{\varepsilon}\right\|_{W^{1, \infty}}\right)$ to close the a priori $L^{2}\left(\Omega ; H^{s}\right)$ estimate for $u_{\varepsilon}$. Then we can go along the lines as we prove Lemma 3.1 to find that for each fixed $\varepsilon$, if $T_{\varepsilon}<\infty$, then $\lim _{\sup _{t \rightarrow T_{\varepsilon}}}\left\|u_{\varepsilon}(t)\right\|_{W^{1, \infty}}=\infty$. Due to the cut-off in (3.5), for a.e. $\omega \in \Omega,\left\|u_{\varepsilon}(t)\right\|_{W^{1, \infty}}$ is always bounded and hence $u_{\varepsilon}$ is actually a global in time solution, that is, $u_{\varepsilon} \in C\left([0, \infty), H^{s}\right) \mathbb{P}-$ a.s. We remark here that the global existence of $u_{\varepsilon}$ is necessary in our framework due to the lack of lifespan estimate in the stochastic setting. Otherwise we will have to prove $\mathbb{P}\left\{\inf _{\varepsilon>0} T_{\varepsilon}>0\right\}=1$, which is not clear in general.

2. (Pathwise solution to the cut-off problem in $H^{s}$ with $s>3$ ) We pass to the limit $\varepsilon \rightarrow 0$. By applying the stochastic compactness arguments from Prokhorov's and Skorokhod's theorems, we obtain the almost sure convergence for a new approximate solution $\left(\widetilde{u_{\varepsilon}}, \widetilde{W}_{\varepsilon}\right)$ defined on a new probability space. By virtue of a refined martingale representation theorem [36, Theorem A.1], we may send $\varepsilon \rightarrow 0$ in $\left(\widetilde{u_{\varepsilon}}, \widetilde{W}_{\varepsilon}\right)$ to build a global martingale solution in $H^{s}$ with $s>3$ to the cut-off problem. Finally, since $F$ satisfies the estimates as in Lemma A.5 and $h$ satisfies Assumption $A_{1}$, one can obtain the pathwise uniqueness easily. Then the Gyöngy-Krylov characterization [30] of the convergence in probability can be applied here to prove the convergence of the original approximate solutions. For more details, we refer to [58,59].

3. (Remove the cut-off and extend the range of $s$ to $s>3 / 2$ ) When $u_{0} \in L^{\infty}\left(\Omega, H^{s}\right)$ with $s>3 / 2$, by mollifying the initial data, we obtain a sequence of regular solutions $\left\{u_{n}\right\}_{n \in \mathbb{N}}$ to $(1.5)$. Motivated by [28,58], one can prove that there is a subsequence (still denoted by $u_{n}$ ) such that for some almost surely positive stopping time $\tau$,

$$
\lim _{n \rightarrow \infty} \sup _{t \in[0, \tau]}\left\|u_{n}-u\right\|_{H^{s}}=0 \mathbb{P}-\text { a.s. }
$$

and

$$
\sup _{t \in[0, \tau]}\|u\|_{H^{s}} \leq\left\|u_{0}\right\|_{H^{s}}+2 \mathbb{P}-\text { a.s. }
$$

Then we can pass the limit $n \rightarrow \infty$ to prove that $(u, \tau)$ is a solution to (1.5). Besides, using a cutting argument, as in $[3,28,29]$, enables us to remove the $L^{\infty}(\Omega)$ assumption on $u_{0}$. More precisely, when $\mathbb{E}\left\|u_{0}\right\|_{H^{s}}^{2}<\infty$, we consider the decomposition

$$
\Omega_{m}=\left\{m-1 \leq\left\|u_{0}\right\|_{H^{s}}<m\right\}, m \geq 1 .
$$

Since $\mathbb{E}\left\|u_{0}\right\|_{H^{s}}^{2}<\infty, \bigcup_{m \geq 1} \Omega_{m}$ is a set of full measure and $1=\sum_{m \geq 1} \mathbf{1}_{\Omega_{m}} \mathbb{P}-$ a.s. Therefore we have

$$
u_{0}(\omega, x)=\sum_{m \geq 1} u_{0, m}(\omega, x)=\sum_{m \geq 1} u_{0}(\omega, x) \mathbf{1}_{\Omega_{m}} \mathbb{P}-\text { a.s. }
$$


For each initial value $u_{0, m}$, we let $\left(u_{m}, \tau_{m}\right)$ be the pathwise unique solution to (1.5) satisfying (3.6). Moreover, as $F(0)=0$ and $h(0)=0$ (cf. (2.3)), direct computation shows that

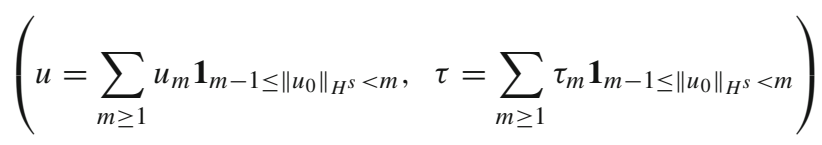

is the unique pathwise solution to (1.5) corresponding to the initial condition $u_{0}$. Since $\left(u_{m}, \tau_{m}\right)$ satisfies (3.6), we have $u(\cdot \wedge \tau) \in C\left([0, \infty) ; H^{s}\right) \mathbb{P}-$ a.s. and

$$
\begin{aligned}
\sup _{t \in[0, \tau]}\|u\|_{H^{s}}^{2} & =\sum_{m=1}^{\infty} \mathbf{1}_{m-1 \leq\left\|u_{0}\right\|_{H^{s}<m}} \sup _{t \in\left[0, \tau_{m}\right]}\left\|u_{m}\right\|_{H^{s}}^{2} \\
& \leq C \sum_{m=1}^{\infty} \mathbf{1}_{m-1 \leq\left\|u_{0}\right\|_{H^{s}}<m}\left(4+\left\|u_{0, m}\right\|_{H^{s}}^{2}\right)=C\left(4+\left\|u_{0}\right\|_{H^{s}}^{2}\right) .
\end{aligned}
$$

Taking expectation in the above inequality, we obtain (2.6). Since the passage from $(u, \tau)$ to a unique maximal pathwise solution $\left(u, \tau^{*}\right)$ in the sense of Definition 2.1 can be carried out as in $[18,28,29,57]$, we omit the details. The proof for Theorem 2.1 is finished.

Remark 3.1 Changing the growth assumption (2.3) to $\|h(t, u)\|_{L_{2}\left(U ; H^{s}\right)} \leq f\left(\|u\|_{H^{s^{\prime}}}\right)(1+$ $\|u\|_{H^{s}}$ ) with $H^{s} \hookrightarrow H^{s^{\prime}} \hookrightarrow W^{1, \infty}$ leads to a criticality statement. One can then go along the lines in Lemma 3.1 to find $\mathbf{1}_{\left\{\tau^{*}<\infty\right\}}=\mathbf{1}_{\left\{\lim \sup _{t \rightarrow \tau^{*}}\|u(t)\|_{H^{s^{\prime}}}=\infty\right\}} \mathbb{P}-$ a.s. Using another cut-off $\chi_{R}\left(\|u\|_{H^{s^{\prime}}}\right)$ in the approximate scheme (3.4) and in (3.5), the other part of the proof also allows us to establish a local existence and uniqueness result. The difference condition (2.4) is essential to guarantee pathwise uniqueness.

\section{Proof for Theorem 2.2}

Now we are going to prove Theorem 2.2. To this end, it suffices to show that if for some $R_{0} \gg 1$, the $R_{0}$-exiting time is strongly stable at the zero solution, then the solution map $u_{0} \mapsto u$ is not uniformly continuous. We restrict our attention to $k \geq 2$ since the case $k=1$, i.e., the stochastic $\mathrm{CH}$ equation, has been obtained in [59].

\subsection{Approximate Solutions and Associated Estimates}

Define the approximate solutions as

$$
u^{l, n}=\ln { }^{-\frac{1}{k}}+n^{-s} \cos \theta \text { and } \theta=n x-l t, n \in \mathbb{Z}^{+},
$$

where

$$
l \in\{-1,1\} \text { if } k \text { is odd; } l \in\{0,1\} \text { if } k \text { is even. }
$$

Substituting $u^{l, n}$ into (1.5), we see that the error $E^{l, n}(t)$ can be defined as

$$
E^{l, n}(t)=u^{l, n}(t)-u^{l, n}(0)+\int_{0}^{t}\left[\left(u^{l, n}\right)^{k} \partial_{x} u^{l, n}+F\left(u^{l, n}\right)\right] \mathrm{d} t^{\prime}-\int_{0}^{t} h\left(t^{\prime}, u^{l, n}\right) \mathrm{d} \mathcal{W} .
$$

Now we analyze the error as follows. 
Lemma 4.1 Let $s>3 / 2$. For $n \gg 1, \delta \in(1 / 2, \min \{s-1,3 / 2\})$ and any $T>0$, there is a $C=C(T)>0$ such that

$$
\mathbb{E} \sup _{t \in[0, T]}\left\|E^{l, n}(t)\right\|_{H^{\delta}}^{2} \leq C n^{-2 r_{s}} .
$$

Here $r_{s}$ is a parameter with

$$
0<r_{s}= \begin{cases}2 s-\delta-\frac{k+1}{k}, & \text { if } \frac{3}{2}<s \leq \frac{2 k+1}{k}, \\ s-\delta+1, & \text { if } s>\frac{2 k+1}{k} .\end{cases}
$$

Proof Since (4.1) implies $l^{k}=l$. This means

$$
\begin{aligned}
& u^{l, n}(t)-u^{l, n}(0)+\int_{0}^{t}\left(u^{l, n}\right)^{k} \partial_{x} u^{l, n} \mathrm{~d} t^{\prime} \\
= & u^{l, n}(t)-u^{l, n}(0)+\int_{0}^{t}\left(\sum_{j=0}^{k}\left(\begin{array}{l}
k \\
j
\end{array}\right)\left(l n^{-\frac{1}{k}}\right)^{k-j} n^{-s j} \cos ^{j} \theta\right)\left(-n^{-s+1} \sin \theta\right) \mathrm{d} t^{\prime} \\
= & \int_{0}^{t}\left(\sum_{j=1}^{k}\left(\begin{array}{l}
k \\
j
\end{array}\right)\left(l n^{-\frac{1}{k}}\right)^{k-j} n^{-s j} \cos ^{j} \theta\right)\left(-n^{-s+1} \sin \theta\right) \mathrm{d} t^{\prime} \triangleq \int_{0}^{t} T_{n, k} \mathrm{~d} t^{\prime} .
\end{aligned}
$$

Then we have

$$
E^{l, n}(t)-\int_{0}^{t}\left[T_{n, k}+F\left(u^{l, n}\right)\right] \mathrm{d} t^{\prime}+\int_{0}^{t} h\left(t^{\prime}, u^{l, n}\right) \mathrm{d} \mathcal{W}=0 .
$$

We first notice that

$$
\begin{aligned}
\left\|T_{n, k}\right\|_{H^{\delta}} & \lesssim \sum_{j=1}^{k} n^{\frac{j}{k}-s j-s}\left\|\cos ^{j} \theta \sin \theta\right\|_{H^{\delta}} \\
& \lesssim \sum_{j=1}^{k} n^{\frac{j}{k}-s j-s-1}\left\|\cos ^{j+1} \theta\right\|_{H^{\delta+1}} \\
& \lesssim \max _{1 \leq j \leq n}\left\{n^{\frac{j}{k}-s j-s+\delta}\right\} \lesssim n^{\frac{1}{k}-2 s+\delta} \lesssim n^{1-2 s+\delta} .
\end{aligned}
$$

Recall that $F(\cdot)$ is given by (1.6). Since $\left(1-\partial_{x x}^{2}\right)^{-1}$ is bounded from $H^{\delta}$ to $H^{\delta+2}$, we can use Lemmas A.6 and A.3 to estimate $\left\|F_{i}\left(u^{l, n}\right)\right\|_{H^{\delta}}(i=1,2,3)$ as follows.

$$
\begin{aligned}
\left\|F_{1}\left(u^{l, n}\right)\right\|_{H^{\delta}} & \lesssim \sum_{j=0}^{k} n^{\frac{j}{k}-s j-s}\left\|\cos ^{j} \theta \sin \theta\right\|_{H^{\delta-2}} \\
& \lesssim \sum_{j=0}^{k} n^{\frac{j}{k}-s j-s-1}\left\|\cos ^{j+1} \theta\right\|_{H^{\delta-1}} \\
& \lesssim \sum_{j=0}^{k} n^{\frac{j}{k}-s j-s-1}\left\|\cos ^{j+1} \theta\right\|_{H^{\delta}} \\
& \lesssim \max _{0 \leq j \leq k}\left\{n^{\frac{j}{k}-s j-s-1+\delta}\right\} \lesssim n^{-s+\delta-1} .
\end{aligned}
$$




$$
\begin{aligned}
\left\|F_{2}\left(u^{l, n}\right)\right\|_{H^{\delta}} & \lesssim \sum_{j=0}^{k-1} n^{\frac{j+1}{k}-s j-2 s+1}\left\|\cos ^{j} \theta \sin ^{2} \theta\right\|_{H^{\delta-1}} \\
& \lesssim \sum_{j=0}^{k-1} n^{\frac{j+1}{k}-s j-2 s+1}\left\|\cos ^{j} \theta \sin ^{2} \theta\right\|_{H^{\delta}} \\
& \lesssim \max _{0 \leq j \leq k-1}\left\{n^{\frac{j+1}{k}-s j-2 s+\delta+1}\right\} \lesssim n^{-2 s+\delta+1+\frac{1}{k}} \\
\left\|F_{3}\left(u^{l, n}\right)\right\|_{H^{\delta}} & \lesssim \sum_{j=0}^{k-2} n^{\frac{j+2}{k}-s j-3 s+2}\left\|\cos ^{j} \theta \sin ^{3} \theta\right\|_{H^{\delta-2}} \\
& \lesssim \sum_{j=0}^{k-2} n^{\frac{j+2}{k}-s j-3 s+2}\left\|\cos ^{j} \theta \sin ^{3} \theta\right\|_{H^{\delta}} \\
& \lesssim \max _{0 \leq j \leq k-2}\left\{n^{\frac{j+2}{k}-s j-3 s+\delta+2}\right\} \lesssim n^{-3 s+\delta+\frac{2}{k}+2} .
\end{aligned}
$$

In the above estimates, we used the fact that $F_{3}(\cdot)$ appears only for $k \geq 2$. Combining this fact, (4.6), (4.7) and (4.8), we have

$$
\left\|F\left(u^{l, n}\right)\right\|_{H^{\delta}} \lesssim \max \left\{n^{1-2 s+\delta}, n^{-3 s+\delta+\frac{2}{k}+2}, n^{-2 s+\delta+\frac{1}{k}+1}, n^{-s+\delta-1}\right\} \lesssim n^{-r_{s}} .
$$

Then, for any $T>0$ and $t \in[0, T]$, by virtue of the Itô formula, we arrive at

$$
\left\|E^{l, n}(t)\right\|_{H^{\delta}}^{2} \leq\left|\left(-2 \int_{0}^{t} h\left(t^{\prime}, u^{l, n}\right) \mathrm{d} \mathcal{W}, E^{l, n}\right)_{H^{\delta}}\right|+\sum_{i=2}^{4} \int_{0}^{t}\left|J_{i}\right| \mathrm{d} t^{\prime},
$$

where

$$
\begin{aligned}
& J_{2}=2\left(D^{\delta} T_{n, k}, D^{\delta} E^{l, n}\right)_{L^{2}}, \\
& J_{3}=2\left(D^{\delta} F\left(u^{l, n}\right), D^{\delta} E^{l, n}\right)_{L^{2}}, \\
& J_{4}=\left\|h\left(t^{\prime}, u^{l, n}\right)\right\|_{L_{2}\left(U, H^{\delta}\right)}^{2} .
\end{aligned}
$$

Taking the supremum with respect to $t \in[0, T]$, using the Burkholder-Davis-Gundy inequality and using (2.5) and (4.9) yield

$$
\begin{aligned}
\mathbb{E} \sup _{t \in[0, T]}\left|\left(-2 \int_{0}^{t} h\left(t^{\prime}, u^{l, n}\right) \mathrm{d} \mathcal{W}, E^{l, n}\right)_{H^{\delta}}\right| & \leq 2 \mathbb{E}\left(\int_{0}^{T}\left\|E^{l, n}(t)\right\|_{H^{\delta}}^{2}\left\|F\left(u^{l, n}\right)\right\|_{H^{\delta}}^{2} \mathrm{~d} t\right)^{\frac{1}{2}} \\
& \leq 2 \mathbb{E}\left(\sup _{t \in[0, T]}\left\|E^{l, n}(t)\right\|_{H^{\delta}}^{2} \int_{0}^{T}\left\|F\left(u^{l, n}\right)\right\|_{H^{\delta}}^{2} \mathrm{~d} t\right)^{\frac{1}{2}} \\
& \leq \frac{1}{2} \mathbb{E} \sup _{t \in[0, T]}\left\|E^{l, n}(t)\right\|_{H^{\delta}}^{2}+C T n^{-2 r_{s}} .
\end{aligned}
$$

By virtue of (4.5) and (4.9), we obtain

$$
\int_{0}^{T} \mathbb{E}\left|J_{2}\right| \mathrm{d} t \leq C \int_{0}^{T} \mathbb{E}\left(\left\|T_{n, k}\right\|_{H^{\delta}}\left\|E^{l, n}(t)\right\|_{H^{\delta}}\right) \mathrm{d} t
$$




$$
\begin{aligned}
& \leq C \int_{0}^{T} \mathbb{E}\left\|T_{n, k}\right\|_{H^{\delta}}^{2} \mathrm{~d} t+C \int_{0}^{T} \mathbb{E}\left\|E^{l, n}(t)\right\|_{H^{\delta}}^{2} \mathrm{~d} t \\
& \leq C T n^{-2 r_{s}}+C \int_{0}^{T} \mathbb{E}\left\|E^{l, n}(t)\right\|_{H^{\delta}}^{2} \mathrm{~d} t, \\
\int_{0}^{T} \mathbb{E}\left|J_{3}\right| \mathrm{d} t & \leq C \int_{0}^{T} \mathbb{E}\left(\left\|F\left(u^{l, n}\right)\right\|_{H^{\delta}}\left\|E^{l, n}(t)\right\|_{H^{\delta}}\right) \mathrm{d} t \\
& \leq C \int_{0}^{T} \mathbb{E}\left\|F\left(u^{l, n}\right)\right\|_{H^{\delta}}^{2} \mathrm{~d} t+C \int_{0}^{T} \mathbb{E}\left\|E^{l, n}(t)\right\|_{H^{\delta}}^{2} \mathrm{~d} t \\
& \leq C T n^{-2 r_{s}}+C \int_{0}^{T} \mathbb{E}\left\|E^{l, n}(t)\right\|_{H^{\delta}}^{2} \mathrm{~d} t,
\end{aligned}
$$

and by (2.5),

$$
\int_{0}^{T} \mathbb{E}\left|J_{4}\right| \mathrm{d} t \leq C \int_{0}^{T} \mathbb{E}\left\|F\left(u^{l, n}\right)\right\|_{H^{\delta}}^{2} \mathrm{~d} t \leq C T n^{-2 r_{s}} .
$$

Collecting the above estimates, we arrive at

$$
\mathbb{E} \sup _{t \in[0, T]}\left\|E^{l, n}(t)\right\|_{H^{\delta}}^{2} \leq C T n^{-2 r_{s}}+C \int_{0}^{T} \mathbb{E} \sup _{t^{\prime} \in[0, t]}\left\|E^{l, n}\left(t^{\prime}\right)\right\|_{H^{\delta}}^{2} \mathrm{~d} t .
$$

Obviously, for each $n \geq 1$ and $l \in\{-1,1\}, \mathbb{E} \sup _{t^{\prime} \in[0, t]}\left\|E^{l, n}\left(t^{\prime}\right)\right\|_{H^{\delta}}^{2}$ is finite. Then we infer from the Grönwall inequality that

$$
\mathbb{E} \sup _{t \in[0, T]}\left\|E^{l, n}(t)\right\|_{H^{\delta}}^{2} \leq C n^{-2 r_{s}}, \quad C=C(T) .
$$

This is the desired result.

\subsection{Construction of Actual Solutions}

Now we consider the following periodic boundary value problem with deterministic initial data $u^{l, n}(0, x)$, i.e.,

$$
\left\{\begin{array}{l}
\mathrm{d} u+\left[u^{k} u_{x}+F(u)\right] \mathrm{d} t=h(t, u) \mathrm{d} \mathcal{W}, \quad t>0, x \in \mathbb{T}, \\
u(0, x)=u^{l, n}(0, x), \quad x \in \mathbb{T} .
\end{array}\right.
$$

Since $h$ satisfies (2.5), we see that (2.3) and (2.4) are also verified. Then Theorem 2.1 yields that for each $n \in \mathbb{N}$, (4.10) has a uniqueness maximal pathwise solution $\left(u_{l, n}, \tau_{l, n}^{*}\right)$.

\subsection{Estimates on the Errors}

Lemma 4.2 Let $s>\frac{3}{2}, \frac{1}{2}<\delta<\min \left\{s-1, \frac{3}{2}\right\}$ and $r_{s}>0$ be given as in Lemma 4.1. For any $R>1$ and $l$ satisfying (4.1), we define

$$
\tau_{l, n}^{R}:=\inf \left\{t \geq 0:\left\|u_{l, n}\right\|_{H^{s}}>R\right\} .
$$

Then for any $T>0$, when $n \gg 1$, we have that for l satisfying (4.1),

$$
\mathbb{E} \sup _{t \in\left[0, T \wedge \tau_{l, n}^{R}\right]}\left\|u^{l, n}-u_{l, n}\right\|_{H^{\delta}}^{2} \leq C n^{-2 r_{s}}, \quad C=C(R, T),
$$


and

$$
\mathbb{E} \sup _{t \in\left[0, T \wedge \tau_{l, n}^{R}\right]}\left\|u^{l, n}-u_{l, n}\right\|_{H^{2 s-\delta}}^{2} \leq C n^{2 s-2 \sigma}, \quad C=C(R, T) .
$$

Proof We first notice that by Lemma A.6, for $l$ satisfying (4.1),

$$
\left\|u^{l, n}(t)\right\|_{H^{s}} \lesssim 1, \quad \forall t>0 .
$$

Let $q=q^{l, n}=\sum_{i=0}^{k}\left(u^{l, n}\right)^{k-i}\left(u_{l, n}\right)^{i}$ and $v=v^{l, n}=u^{l, n}-u_{l, n}$. In view of (4.2), (4.4) and (4.10), we see that

$$
v(t)+\int_{0}^{t}\left[\frac{1}{k+1} \partial_{x}(q v)-F\left(u_{l, n}\right)\right] \mathrm{d} t^{\prime}=-\int_{0}^{t} h\left(t^{\prime}, u_{l, n}\right) \mathrm{d} \mathcal{W}+\int_{0}^{t} T_{n, k} \mathrm{~d} t^{\prime} .
$$

For any $T>0$, we use the Itô formula on $\left[0, T \wedge \tau_{l, n}^{R}\right]$, take the supremum over $t \in\left[0, T \wedge \tau_{l, n}^{R}\right]$ and use the Burkholder-Davis-Gundy inequality with noticing (2.5) to find

$$
\mathbb{E} \sup _{t \in\left[0, T \wedge \tau_{l, n}^{R}\right]}\|v(t)\|_{H^{\delta}}^{2} \leq C \mathbb{E}\left(\int_{0}^{T \wedge \tau_{l, n}^{R}}\left|K_{1}\right|^{2} \mathrm{~d} t\right)^{\frac{1}{2}}+\sum_{i=2}^{5} \mathbb{E} \int_{0}^{T \wedge \tau_{l, n}^{R}}\left|K_{i}\right| \mathrm{d} t,
$$

where

$$
\begin{aligned}
K_{1} & =\|v\|_{H^{\delta}}\left\|F\left(u_{l, n}\right)\right\|_{H^{\delta}}, \\
K_{2} & =2\left(D^{\delta} T_{n, k}, D^{\delta} v\right)_{L^{2}}, \\
K_{3} & =-\frac{2}{k+1}\left(D^{\delta} \partial_{x}[q v], D^{\delta} v\right)_{L^{2}}, \\
K_{4} & =2\left(D^{\delta} F\left(u_{l, n}\right), D^{\delta} v\right)_{L^{2}}, \\
K_{5} & =\left\|h\left(t, u_{l, n}\right)\right\|_{L_{2}\left(U, H^{\delta}\right)}^{2} .
\end{aligned}
$$

We can first infer from Lemma A.5 that

$$
\begin{aligned}
\left\|F\left(u_{l, n}\right)\right\|_{H^{\delta}}^{2} & \lesssim\left(\left\|F\left(u^{l, n}\right)-F\left(u_{l, n}\right)\right\|_{H^{\delta}}+\left\|F\left(u^{l, n}\right)\right\|_{H^{\delta}}\right)^{2} \\
& \lesssim\left(\left\|u^{l, n}\right\|_{H^{s}}+\left\|u_{l, n}\right\|_{H^{s}}\right)^{2}\|v\|_{H^{\delta}}^{2}+\left\|F\left(u^{l, n}\right)\right\|_{H^{\delta}}^{2} .
\end{aligned}
$$

Therefore, applying Lemmas A.4 and A.5, $H^{\delta} \hookrightarrow L^{\infty}$, integrating by part and (4.5), we have

$$
\begin{aligned}
\left|K_{1}\right|^{2} & \lesssim\left(\left\|u^{l, n}\right\|_{H^{s}}+\left\|u_{l, n}\right\|_{H^{s}}\right)^{2}\|v\|_{H^{\delta}}^{4}+\left\|F\left(u^{l, n}\right)\right\|_{H^{\delta}}^{2}\|v\|_{H^{\delta}}^{2}, \\
\left|K_{2}\right| & \lesssim\left\|T_{n, k}\right\|_{H^{\delta}}^{2}+\|v\|_{H^{\delta}}^{2} \lesssim n^{-2 r_{s}}+\|v\|_{H^{\delta}}^{2}, \\
\left|K_{3}\right| & \lesssim\|q\|_{H^{s}}\|v\|_{H^{\delta}}^{2}+\left\|q_{x}\right\|_{L^{\infty}}\|v\|_{H^{\delta}}^{2} \lesssim\|q\|_{H^{s}}\|v\|_{H^{\delta}}^{2}, \\
\left|K_{4}\right| & \lesssim\left(\left\|u^{l, n}\right\|_{H^{s}}+\left\|u_{l, n}\right\|_{H^{s}}\right)\|v\|_{H^{\delta}}^{2}+\left\|F\left(u^{l, n}\right)\right\|_{H^{\delta}}^{2}+\|v\|_{H^{\delta}}^{2},
\end{aligned}
$$

and

$$
\left|K_{5}\right| \lesssim\left(\left\|u^{l, n}\right\|_{H^{s}}+\left\|u_{l, n}\right\|_{H^{s}}\right)^{2}\|v\|_{H^{\delta}}^{2}+\left\|F\left(u^{l, n}\right)\right\|_{H^{\delta}}^{2}
$$


By virtue of Lemmas A.5, (4.9), (4.11) and (4.14), we have

$$
\begin{aligned}
& C \mathbb{E}\left(\int_{0}^{T \wedge \tau_{l, n}^{R}}\left|K_{1}\right|^{2} \mathrm{~d} t\right)^{\frac{1}{2}} \\
& \leq C \mathbb{E}\left(\sup _{t \in\left[0, T \wedge \tau_{l, n}^{R}\right]}\|v\|_{H^{\delta}}^{2} \int_{0}^{T \wedge \tau_{l, n}^{R}}\left(\left\|u^{l, n}\right\|_{H^{s}}+\left\|u_{l, n}\right\|_{H^{s}}\right)^{2}\|v\|_{H^{\delta}}^{2} \mathrm{~d} t\right)^{\frac{1}{2}} \\
& +C \mathbb{E}\left(\sup _{t \in\left[0, T \wedge \tau_{l, n}^{R}\right]}\|v\|_{H^{\delta}}^{2} \int_{0}^{T \wedge \tau_{l, n}^{R}}\left\|F\left(u^{l, n}\right)\right\|_{H^{\delta}}^{2} \mathrm{~d} t\right)^{\frac{1}{2}} \\
& \leq \frac{1}{2} \mathbb{E} \sup _{t \in\left[0, T \wedge \tau_{l, n}^{R}\right]}\|v\|_{H^{\delta}}^{2}+C_{R} \mathbb{E} \int_{0}^{T \wedge \tau_{l, n}^{R}}\|v(t)\|_{H^{\delta}}^{2} \mathrm{~d} t+C \mathbb{E} \int_{0}^{T \wedge \tau_{l, n}^{R}}\left\|F\left(u^{l, n}\right)\right\|_{H^{\delta}}^{2} \mathrm{~d} t \\
& \leq \frac{1}{2} \mathbb{E} \sup _{t \in\left[0, T \wedge \tau_{l, n}^{R}\right]}\|v\|_{H^{\delta}}^{2}+C_{R} \mathbb{E} \int_{0}^{T} \sup _{t^{\prime} \in\left[0, t \wedge \tau_{l, n}^{R}\right]}\left\|v\left(t^{\prime}\right)\right\|_{H^{\delta}}^{2} \mathrm{~d} t+C T n^{-2 r_{s}}, \\
& \mathbb{E} \int_{0}^{T \wedge \tau_{l, n}^{R}}\left|K_{2}\right|+\left|K_{4}\right|+\left|K_{5}\right| \mathrm{d} t \leq C T n^{-2 r_{s}}+C_{R} \int_{0}^{T} \mathbb{E} \sup _{t^{\prime} \in\left[0, t \wedge \tau_{l, n}^{R}\right]}\left\|v\left(t^{\prime}\right)\right\|_{H^{\delta}}^{2} \mathrm{~d} t,
\end{aligned}
$$

and

$$
\mathbb{E} \int_{0}^{T \wedge \tau_{l, n}^{R}}\left|K_{3}\right| \mathrm{d} t \leq C_{R} \int_{0}^{T} \mathbb{E} \sup _{t^{\prime} \in\left[0, t \wedge \tau_{l, n}^{R}\right]}\left\|v\left(t^{\prime}\right)\right\|_{H^{\delta}}^{2} \mathrm{~d} t
$$

Consequently,

$$
\mathbb{E} \sup _{t \in\left[0, T \wedge \tau_{l, n}^{R}\right]}\|v(t)\|_{H^{\delta}}^{2} \leq C T n^{-2 r_{s}}+C_{R} \int_{0}^{T} \mathbb{E} \sup _{t^{\prime} \in\left[0, t \wedge \tau_{l, n}^{R}\right]}\left\|v\left(t^{\prime}\right)\right\|_{H^{\delta}}^{2} \mathrm{~d} t .
$$

Using the Grönwall inequality, we have

$$
\mathbb{E} \sup _{t \in\left[0, T \wedge \tau_{l, n}^{R}\right]}\|v(t)\|_{H^{\delta}}^{2} \leq C n^{-2 r_{s}}, \quad C=C(R, T),
$$

which is (4.12). For (4.13), we first notice that $u_{l, n}$ is the unique solution to (4.10) with $2 s-\delta>3 / 2$. For each fixed $n \in \mathbb{Z}^{+}$, we can go along the lines as we derive (3.3) with using (4.11) to find

$$
\mathbb{E} \sup _{t \in\left[0, T \wedge \tau_{l, n}^{R}\right]}\left\|u_{l, n}(t)\right\|_{H^{2 s-\delta}}^{2} \leq C \mathbb{E}\left\|u^{l, n}(0)\right\|_{H^{2 s-\delta}}^{2}+C_{R} \int_{0}^{T}\left(\mathbb{E} \sup _{t^{\prime} \in\left[0, t \wedge \tau_{l, n}^{R}\right]}\left\|u_{l, n}\left(t^{\prime}\right)\right\|_{H^{2 s-\delta}}^{2}\right) \mathrm{d} t .
$$

From (4.15), we can use the Grönwall inequality and Lemma A.6 to infer

$$
\mathbb{E} \sup _{t \in\left[0, T \wedge \tau_{l, n}^{R}\right]}\left\|u_{l, n}(t)\right\|_{H^{2 s-\delta}}^{2} \leq C \mathbb{E}\left\|u^{l, n}(0)\right\|_{H^{2 s-\delta}}^{2} \leq C n^{2 s-2 \delta}, \quad C=C(R, T) .
$$


By Lemma A.6 again, we have that for some $C=C(R, T)$ and $l$ satisfying (4.1),

$$
\mathbb{E} \sup _{t \in\left[0, T \wedge \tau_{l, n}^{R}\right]}\|v\|_{H^{2 s-\delta}}^{2} \leq C \mathbb{E} \sup _{t \in\left[0, T \wedge \tau_{l, n}^{R}\right]}\left\|u_{l, n}\right\|_{H^{2 s-\delta}}^{2}+C \mathbb{E} \sup _{t \in\left[0, T \wedge \tau_{l, n}^{R}\right]}\left\|u^{l, n}\right\|_{H^{2 s-\delta}}^{2} \leq C n^{2 s-2 \delta},
$$

which is (4.13).

\subsection{Final Proof for Theorem 2.2}

To begin with, we will show that if the exiting time of the zero solution is strongly stable, then (2.8)-(2.11) are satisfied.

Lemma 4.3 If for some $R_{0} \gg 1$, the $R_{0}$-exiting time of the zero solution to (1.5) is strongly stable, then for $l$ satisfying (4.1) and $\tau_{l, n}^{R_{0}}$ given in (4.11), we have

$$
\lim _{n \rightarrow \infty} \tau_{l, n}^{R_{0}}=\infty \mathbb{P}-\text { a.s. }
$$

Proof We notice that for all $s^{\prime}<s, \lim _{n \rightarrow \infty}\left\|u^{l, n}(0)\right\|_{H^{s^{\prime}}}=\lim _{n \rightarrow \infty}\left\|u_{l, n}(0)-0\right\|_{H^{s^{\prime}}}=0$. Since the unique solution with zero initial data to (1.5) is zero and the $R_{0}$-exiting time of the zero solution is $\infty$, we see that (4.16) holds true provided the $R_{0}$-exiting time of the zero solution to (1.5) is strongly stable.

Proof for Theorem 2.2 For each $n>1$, for $l$ satisfying (4.1) and for the fixed $R_{0} \gg 1$, Lemma A.6 and (4.11) give us $\mathbb{P}\left\{\tau_{l, n}^{R_{0}}>0\right\}=1$ and Lemma 4.3 implies (2.8). Besides, Theorem 2.1 and (4.11) show that $u_{l, n} \in C\left(\left[0, \tau_{l, n}^{R_{0}}\right] ; H^{s}\right) \mathbb{P}-$ a.s. and (2.9) holds true. By virtue of the interpolation inequality, we have

$$
\begin{aligned}
& \mathbb{E} \sup _{t \in\left[0, T \wedge \tau_{l, n}^{R_{0}}\right]}\left\|u^{l, n}-u_{l, n}\right\|_{H^{s}} \\
& \leq C\left(\mathbb{E} \sup _{t \in\left[0, T \wedge \tau_{l, n}^{R_{0}}\right]}\left\|u^{l, n}-u_{l, n}\right\|_{H^{\delta}}\right)^{\frac{1}{2}}\left(\sup _{t \in\left[0, T \wedge \tau_{l, n}^{R_{0}}\right]}\left\|u^{l, n}-u_{l, n}\right\|_{H^{2 s-\delta}}\right)^{\frac{1}{2}}
\end{aligned}
$$

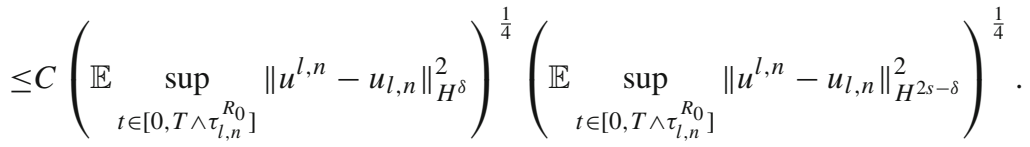

For any $T>0$, combining Lemma 4.2 and the above estimate yields

$$
\mathbb{E} \sup _{t \in\left[0, T \wedge \tau_{l, n}^{R_{0}}\right]}\left\|u^{l, n}-u_{l, n}\right\|_{H^{s}} \lesssim n^{-\frac{1}{4} \cdot 2 r_{s}} \cdot n^{\frac{1}{4} \cdot(2 s-2 \delta)}=n^{r_{s}^{\prime}},
$$

for some $C=C\left(R_{0}, T\right)$ and $l$ satisfying (4.1). Recalling $k \geq 2$ and (4.3), we have

$$
0>r_{s}^{\prime}=-r_{s} \cdot \frac{1}{2}+(s-\delta) \cdot \frac{1}{2}= \begin{cases}-\frac{s}{2}+\frac{k+1}{2 k}, & \text { if } \frac{3}{2}<s \leq \frac{2 k+1}{k}, \\ -\frac{1}{2}, & \text { if } s>\frac{2 k+1}{k} .\end{cases}
$$

Therefore for $l$ satisfying (4.1),

$$
\lim _{n \rightarrow \infty} \mathbb{E} \sup _{t \in\left[0, T \wedge \tau_{l, n}^{R_{0}}\right]}\left\|u^{l, n}-u_{l, n}\right\|_{H^{s}}=0 .
$$


For odd $k,(2.10)$ is given by

$$
\left\|u_{-1, n}(0)-u_{1, n}(0)\right\|_{H^{s}}=\left\|u^{-1, n}(0)-u^{1, n}(0)\right\|_{H^{s}} \lesssim n^{-1 / k} \rightarrow 0, \text { as } n \rightarrow \infty .
$$

For even $k,(2.10)$ is given by

$$
\left\|u_{0, n}(0)-u_{1, n}(0)\right\|_{H^{s}}=\left\|u^{0, n}(0)-u^{1, n}(0)\right\|_{H^{s}} \lesssim n^{-1 / k} \rightarrow 0, \text { as } n \rightarrow \infty .
$$

When $k$ is odd, we use (4.18) to find that for any $T>0$,

$$
\begin{aligned}
& \liminf _{n \rightarrow \infty} \sup _{t \in\left[0, T \wedge \tau_{-1, n}^{R_{0}} \wedge \tau_{1, n}^{R_{0}}\right]}\left\|u_{-1, n}(t)-u_{1, n}(t)\right\|_{H^{s}} \\
& \geq \liminf _{n \rightarrow \infty} \mathbb{E} \sup _{t \in\left[0, T \wedge \tau_{-1, n}^{R_{0}} \wedge \tau_{1, n}^{R_{0}}\right]}\left\|u^{-1, n}(t)-u^{1, n}(t)\right\|_{H^{s}} \\
& -\lim _{n \rightarrow \infty} \mathbb{E} \sup _{t \in\left[0, T \wedge \tau_{-1, n}^{R_{0}} \wedge \tau_{1, n}^{R_{0}}\right]}\left\|u^{-1, n}(t)-u_{-1, n}(t)\right\|_{H^{s}} \\
& -\lim _{n \rightarrow \infty} \mathbb{E} \sup _{t \in\left[0, T \wedge \tau_{-1, n}^{R_{0}} \wedge \tau_{1, n}^{R_{0}}\right]}\left\|u^{1, n}(t)-u_{1, n}(t)\right\|_{H^{s}} \\
& \gtrsim \liminf _{n \rightarrow \infty} \sup _{t \in\left[0, T \wedge \tau_{-1, n}^{R_{0}} \wedge \tau_{1, n}^{R_{0}}\right]}\left\|u^{-1, n}(t)-u^{1, n}(t)\right\|_{H^{s}} \\
& \gtrsim \liminf _{n \rightarrow \infty} \sup _{t \in\left[0, T \wedge \tau_{-1, n}^{R_{0}} \wedge \tau_{1, n}^{R_{0}}\right]}\left\|-2 n^{-1}+n^{-s} \cos (n x+t)-n^{-s} \cos (n x-t)\right\|_{H^{s}} \\
& \gtrsim \liminf _{n \rightarrow \infty} \sup _{t \in\left[0, T \wedge \tau_{-1, n}^{R_{0}} \wedge \tau_{1, n}^{R_{0}}\right]}\left(n^{-s}\|\sin (n x)\|_{H^{s}}|\sin t|-\left\|2 n^{-1}\right\|_{H^{s}}\right) \gtrsim \sup _{t \in[0, T]}|\sin t|,
\end{aligned}
$$

where we have used Fatou's lemma. By

$$
\left(\mathbb{E} \sup _{t \in\left[0, T \wedge \tau_{-1, n}^{R_{0}} \wedge \tau_{1, n}^{R_{0}}\right]}\left\|u_{-1, n}(t)-u_{1, n}(t)\right\|_{H^{s}}^{2}\right)^{\frac{1}{2}} \geq \mathbb{E} \sup _{t \in\left[0, T \wedge \tau_{-1, n}^{R_{0}} \wedge \tau_{1, n}^{R_{0}}\right]}\left\|u_{-1, n}(t)-u_{1, n}(t)\right\|_{H^{s}}
$$

and (4.19), we obtain (2.11). Similarly, we can also prove (2.11) when $k$ is even. The proof is therefore completed.

\section{Global Existence}

In this section, we study the global existence and the blow-up of solutions to (1.8), and estimate the associated probabilities. Motivated by [29,57,58], we introduce the following Girsanov type transform

$$
v=\frac{1}{\beta(\omega, t)} u, \quad \beta(\omega, t)=\mathrm{e}^{\int_{0}^{t} b\left(t^{\prime}\right) \mathrm{d} W_{t^{\prime}}-\int_{0}^{t} \frac{b^{2}\left(t^{\prime}\right)}{2} \mathrm{~d} t^{\prime}} .
$$

Proposition 5.1 Let $s>3 / 2$ and $h(t, u)=b(t) u$ such that $b(t)$ satisfies Assumption $A_{3}$. Let $\mathcal{S}=\left(\Omega, \mathcal{F}, \mathbb{P},\left\{\mathcal{F}_{t}\right\}_{t \geq 0}, W\right)$ be fixed in advance. If $u_{0}(\omega, x)$ is an $H^{s}$-valued $\mathcal{F}_{0}$-measurable random variable with $\mathbb{E}\left\|u_{0}\right\|_{H^{s}}^{2}<\infty$ and $\left(u, \tau^{*}\right)$ is the corresponding unique maximal 
pathwise solution to (1.8), then for $t \in\left[0, \tau^{*}\right)$, the process $v$ given in (5.1) solves

$$
\left\{\begin{array}{l}
v_{t}+\beta^{k} v^{k} v_{x}+\beta^{k} F(v)=0, \quad t>0, x \in \mathbb{T}, \\
v(\omega, 0, x)=u_{0}(\omega, x), x \in \mathbb{T} .
\end{array}\right.
$$

Moreover, one has $v \in C\left(\left[0, \tau^{*}\right) ; H^{s}\right) \bigcap C^{1}\left(\left[0, \tau^{*}\right) ; H^{s-1}\right) \mathbb{P}-$ a.s. and, if $s>3$, then

$$
\mathbb{P}\left\{\|v(t)\|_{H^{1}}=\left\|u_{0}\right\|_{H^{1}}\right\}=1 .
$$

Proof Since $b(t)$ satisfies Assumption $A_{3}, h(t, u)=b(t) u$ satisfies Assumption $A_{1}$. Consequently, Theorem 2.1 implies that (1.8) has a unique maximal pathwise solution $\left(u, \tau^{*}\right)$. Direct computation shows

$$
\mathrm{d} v=\left(-\beta^{k} v^{k} v_{x}-\beta^{k} F(v)\right) \mathrm{d} t .
$$

Since $v(0)=u_{0}(\omega, x)$, we see that $v$ satisfies (5.2). Moreover, Theorem 2.1 implies $u \in$ $C\left(\left[0, \tau^{*}\right) ; H^{s}\right) \mathbb{P}-$ a.s., so $v \in C\left(\left[0, \tau^{*}\right) ; H^{s}\right) \mathbb{P}-$ a.s. Besides, from Lemma A.5 and $(5.2)_{1}$, we see that for a.e. $\omega \in \Omega, v_{t}=-\beta^{k} v^{k} v_{x}-\beta^{k} F(v) \in C\left(\left[0, \tau^{*}\right) ; H^{s-1}\right)$. Hence $v \in C^{1}\left(\left[0, \tau^{*}\right) ; H^{s-1}\right) \mathbb{P}-$ a.s. Notice that if $s>3,(5.2)_{1}$ is equivalent to

$$
v_{t}-v_{x x t}+(k+2) \beta^{k} v^{k} v_{x}=(k+1) \beta^{k} v^{k-1} v_{x} v_{x x}+\beta^{k} v^{k} v_{x x x}, \quad k \geq 1 .
$$

Multiplying both sides of the above equation by $v$ and then integrating the resulting equation on $x \in \mathbb{T}$ with noticing that $(k+1) v^{k} v_{x} v_{x x}+v^{k+1} v_{x x x}=\partial_{x}\left(v^{k+1} v_{x x}\right)$, we see that for a.e. $\omega \in \Omega$ and for all $t>0$

$$
\frac{\mathrm{d}}{\mathrm{d} t} \int_{\mathbb{T}}\left(v^{2}+v_{x}^{2}\right) \mathrm{d} x=0
$$

which implies (5.3).

\subsection{Global Existence: Case 1}

Now we prove the first global existence result.

Proof for Theorem 2.3 To begin with, we apply the operator $D^{s}$ to (5.4), multiply both sides of the resulting equation by $D^{s} v$ and integrate over $\mathbb{T}$ to obtain that for a.e. $\omega \in \Omega$,

$$
\frac{1}{2} \frac{\mathrm{d}}{\mathrm{d} t}\|v(t)\|_{H^{s}}^{2}=-\beta^{k}(\omega, t) \int_{\mathbb{T}} D^{s} v \cdot D^{s}\left[v^{k} v_{x}\right] \mathrm{d} x-\beta^{k}(\omega, t) \int_{\mathbb{T}} D^{s} v \cdot D^{s} F(v) \mathrm{d} x .
$$

Using Lemmas A.2 and A.5, we conclude that there is a $C=C(s)>1$ such that for a.e. $\omega \in \Omega$,

$$
\frac{\mathrm{d}}{\mathrm{d} t}\|v(t)\|_{H^{s}}^{2} \leq C \beta^{k}(t)\|v\|_{W^{1, \infty}}^{k}\|v\|_{H^{s}}^{2}
$$

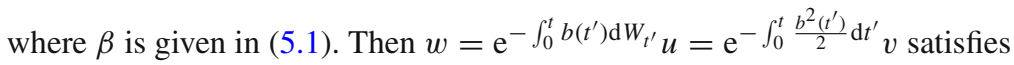
$\frac{\mathrm{d}}{\mathrm{d} t}\|w(t)\|_{H^{s}}+\frac{b^{2}(t)}{2}\|w(t)\|_{H^{s}} \leq C \alpha^{k}(\omega, t)\|w(t)\|_{W^{1, \infty}}^{k}\|w(t)\|_{H^{s}}, \quad \alpha(\omega, t)=\mathrm{e}^{\int_{0}^{t} b\left(t^{\prime}\right) \mathrm{d} W_{t^{\prime}}}$. Let $R>1$ and $\lambda_{1}>2$. Assume $\left\|u_{0}\right\|_{H^{s}}<\frac{1}{R K}\left(\frac{b_{*}}{C \lambda_{1}}\right)^{1 / k}$ almost surely. Define

$$
\tau_{1}(\omega)=\inf \left\{t>0: \alpha^{k}(\omega, t)\|w\|_{W^{1, \infty}}^{k}=\|u\|_{W^{1, \infty}}^{k}>\frac{b^{2}(t)}{C \lambda_{1}}\right\} .
$$


Notice that $\|u(0)\|_{W^{1, \infty}}^{k} \leq K^{k}\|u(0)\|_{H^{s}}^{k}<\frac{b_{*}}{C \lambda_{1}}$. Therefore we have $\mathbb{P}\left\{\tau_{1}>0\right\}=1$, and for $t \in\left[0, \tau_{1}\right)$,

$$
\frac{\mathrm{d}}{\mathrm{d} t}\|w(t)\|_{H^{s}}+\frac{\left(\lambda_{1}-2\right) b^{2}(t)}{2 \lambda_{1}}\|w(t)\|_{H^{s}} \leq 0 .
$$

The above inequality and $w=\mathrm{e}^{-\int_{0}^{t} b\left(t^{\prime}\right) \mathrm{d} W_{t^{\prime}}} u$ imply that for a.e. $\omega \in \Omega$, for any $\lambda_{2}>\frac{2 \lambda_{1}}{\lambda_{1}-2}$ and for $t \in\left[0, \tau_{1}\right)$,

$$
\begin{aligned}
\|u(t)\|_{H^{s}} & \leq\left\|w_{0}\right\|_{H^{s}} \mathrm{e}^{\int_{0}^{t} b\left(t^{\prime}\right) \mathrm{d} W_{t^{\prime}}-\int_{0}^{t} \frac{\left(\lambda_{1}-2\right) b^{2}\left(t^{\prime}\right)}{2 \lambda_{1}} \mathrm{~d} t^{\prime}} \\
& =\left\|u_{0}\right\|_{H^{s}} \mathrm{e}^{\int_{0}^{t} b\left(t^{\prime}\right) \mathrm{d} W_{t^{\prime}}-\int_{0}^{t} \frac{b^{2}\left(t^{\prime}\right)}{\lambda_{2}} \mathrm{~d} t^{\prime}} \mathrm{e}^{-\frac{\left(\left(\lambda_{1}-2\right) \lambda_{2}-2 \lambda_{1}\right)}{2 \lambda_{1} \lambda 2} \int_{0}^{t} b^{2}\left(t^{\prime}\right) \mathrm{d} t^{\prime}}
\end{aligned}
$$

Define the stopping time

$$
\tau_{2}=\tau_{2}(\omega)=\inf \left\{t>0: \mathrm{e}^{\int_{0}^{t} b\left(t^{\prime}\right) \mathrm{d} W_{t^{\prime}}-\int_{0}^{t} \frac{b^{2}\left(t^{\prime}\right)}{\lambda_{2}} \mathrm{~d} t^{\prime}}>R\right\} .
$$

Notice that $\mathbb{P}\left\{\tau_{2}>0\right\}=1$. From (5.7), we have that almost surely

$$
\begin{aligned}
\|u(t)\|_{H^{s}} & <\frac{1}{R K}\left(\frac{b_{*}}{C \lambda_{1}}\right)^{1 / k} \times R \times \mathrm{e}^{-\frac{\left(\left(\lambda_{1}-2\right) \lambda_{2}-2 \lambda_{1}\right)}{2 \lambda_{1} \lambda 2} \int_{0}^{t} b^{2}\left(t^{\prime}\right) \mathrm{d} t^{\prime}} \\
& =\frac{1}{K}\left(\frac{b_{*}}{C \lambda_{1}}\right)^{1 / k} \mathrm{e}^{-\frac{\left(\left(\lambda_{1}-2\right) \lambda_{2}-2 \lambda_{1}\right)}{2 \lambda_{1} \lambda 2} \int_{0}^{t} b^{2}\left(t^{\prime}\right) \mathrm{d} t^{\prime}} \leq \frac{1}{K}\left(\frac{b_{*}}{C \lambda_{1}}\right)^{1 / k}, \quad t \in\left[0, \tau_{1} \wedge \tau_{2}\right) .
\end{aligned}
$$

Combining (5.9) and (5.6), we find that

$$
\mathbb{P}\left\{\tau_{1} \geq \tau_{2}\right\}=1
$$

Therefore it follows from (5.9) that

$$
\mathbb{P}\left\{\|u(t)\|_{H^{s}}<\frac{1}{K}\left(\frac{b_{*}}{C \lambda_{1}}\right)^{1 / k} \mathrm{e}^{-\frac{\left(\left(\lambda_{1}-2\right) \lambda_{2}-2 \lambda_{1}\right)}{2 \lambda_{1} \lambda 2} \int_{0}^{t} b^{2}\left(t^{\prime}\right) \mathrm{d} t^{\prime}} \text { for all } t>0\right\} \geq \mathbb{P}\left\{\tau_{2}=+\infty\right\} .
$$

We apply (ii) in Lemma A.7 to find that

$$
\mathbb{P}\left\{\tau_{2}=+\infty\right\}>1-\left(\frac{1}{R}\right)^{2 / \lambda_{2}},
$$

which completes the proof.

\subsection{Global Existence: Case 2}

Now we prove Theorem 2.4. Let $\beta(\omega, t)$ be given in (5.1). From Proposition 5.1, we see that for a.e. $\omega \in \Omega, v(\omega, t, x)$ solves (5.2) on $\left[0, \tau^{*}\right)$. Moreover, since $H^{s} \hookrightarrow C^{2}$ for $s>3 / 2$, we have $v, v_{x} \in C^{1}\left(\left[0, \tau^{*}\right) \times \mathbb{T}\right)$. Then for a.e. $\omega \in \Omega$, for any $x \in \mathbb{T}$, the problem

$$
\left\{\begin{array}{l}
\frac{d q(\omega, t, x)}{d t}=\beta^{k}(\omega, t) v^{k}(\omega, t, q(\omega, t, x)), \quad t \in\left[0, \tau^{*}\right) \\
q(\omega, 0, x)=x, \quad x \in \mathbb{T}
\end{array}\right.
$$


has a unique solution $q(\omega, t, x)$ such that $q(\omega, t, x) \in C^{1}\left(\left[0, \tau^{*}\right) \times \mathbb{T}\right)$ almost surely. Moreover, differentiating (5.11) with respect to $x$ yields that for a.e. $\omega \in \Omega$,

$$
\left\{\begin{array}{l}
\frac{d q_{x}}{d t}=k \beta^{k}(\omega, t) v^{k-1} v_{x} q_{x}, \quad t \in\left[0, \tau^{*}\right), \\
q_{x}(\omega, 0, x)=1, \quad x \in \mathbb{T}
\end{array}\right.
$$

For a.e. $\omega \in \Omega$, we solve the above equation to obtain

$$
q_{x}(\omega, t, x)=\exp \left(\int_{0}^{t} k \beta^{k}\left(\omega, t^{\prime}\right) v^{k-1} v_{x}\left(\omega, t^{\prime}, q\left(\omega, t^{\prime}, x\right)\right) \mathrm{d} t^{\prime}\right) .
$$

Thus for all $(t, x) \in\left[0, \tau^{*}\right) \times \mathbb{T}$, we find $q_{x}>0$ almost surely.

Lemma 5.1 Let $s>3, V_{0}(\omega, x)=\left(1-\partial_{x x}^{2}\right) u_{0}(\omega, x)$ and $V(\omega, t, x)=v(\omega, t, x)-$ $v_{x x}(\omega, t, x)$, where $v(\omega, t, x)$ solves $(5.2)$ on $\left[0, \tau^{*}\right) \mathbb{P}-$ a.s. Then for all $(t, x) \in\left[0, \tau^{*}\right) \times \mathbb{T}$,

$$
\operatorname{sign}(v)=\operatorname{sign}(V)=\operatorname{sign}\left(V_{0}\right) \mathbb{P}-\text { a.s. }
$$

Proof We first notice that (5.2) 1 is equivalent to (5.5). Therefore for a.e. $\omega \in \Omega, V=v-v_{x x}$ satisfies

$$
V_{t}+\beta^{k} v^{k} V_{x}+(k+1) \beta^{k} V v^{k-1} v_{x}=0 .
$$

Thus for a.e. $\omega \in \Omega$, we have

$$
\begin{aligned}
\frac{\mathrm{d}}{\mathrm{d} t} & {\left[V(\omega, t, q(\omega, t, x)) q_{x}^{\frac{k+1}{k}}(\omega, t, x)\right] } \\
& =V_{t} q_{x}^{\frac{k+1}{k}}+V_{x} q_{t} q_{x}^{\frac{k+1}{k}}+\frac{k+1}{k} V q_{x}^{\frac{k+1}{k}-1} q_{x t} \\
& =q_{x}^{\frac{k+1}{k}}\left[V_{t}+V_{x}\left(\beta^{k} v^{k}\right)+\frac{k+1}{k} V q_{x}^{-1}\left(k \beta^{k} v^{k-1} v_{x} q_{x}\right)\right] \\
& =q_{x}^{\frac{k+1}{k}}\left[V_{t}+\beta^{k} v^{k} V_{x}+(k+1) \beta^{k} V v^{k-1} v_{x}\right]=0 .
\end{aligned}
$$

Notice that $q_{x}(\omega, 0, x)=1$ and $q_{x}>0$ almost surely. Then we have that $\operatorname{sign}(V)=\operatorname{sign}\left(V_{0}\right)$ $\mathbb{P}-$ a.s. Since $v=G_{\mathbb{T}} * V$ with $G_{\mathbb{T}}>0$ given in $(1.7)$, we have $\operatorname{sign}(v)=\operatorname{sign}(V) \mathbb{P}-$ a.s.

Lemma 5.2 Let all the conditions as in the statement of Proposition 5.1 hold true. If

$$
\mathbb{P}\left\{V_{0}(\omega, x)>0, \forall x \in \mathbb{T}\right\}=p, \mathbb{P}\left\{V_{0}(\omega, x)<0, \forall x \in \mathbb{T}\right\}=q,
$$

for some $p, q \in[0,1]$, then the maximal pathwise solution $u$ to (1.8) satisfies

$$
\mathbb{P}\left\{\left\|u_{x}(\omega, t)\right\|_{L^{\infty}} \leq\|u(\omega, t)\|_{L^{\infty}} \lesssim \beta(\omega, t)\left\|u_{0}\right\|_{H^{1}}, \forall t \in\left[0, \tau^{*}\right)\right\} \geq p+q .
$$

Proof It is easy to see that for a.e. $\omega \in \Omega$ and for all $(t, x) \in\left[0, \tau^{*}\right) \times \mathbb{T}$,

$$
\begin{aligned}
& {\left[v+v_{x}\right](\omega, t, x)=\frac{1}{2 \sinh (\pi)} \int_{0}^{2 \pi} \mathrm{e}^{\left(x-y-2 \pi\left[\frac{x-y}{2 \pi}\right]-\pi\right)} V(\omega, t, y) \mathrm{d} y,} \\
& {\left[v-v_{x}\right](\omega, t, x)=\frac{1}{2 \sinh (\pi)} \int_{0}^{2 \pi} \mathrm{e}^{\left(y-x+2 \pi\left[\frac{x-y}{2 \pi}\right]+\pi\right)} V(\omega, t, y) \mathrm{d} y .}
\end{aligned}
$$


Then one can employ (5.12), (5.13) and Lemma 5.1 to obtain that for a.e. $\omega \in \Omega$ and for all $(t, x) \in\left[0, \tau^{*}\right) \times \mathbb{T}$,

$$
\begin{cases}-v(\omega, t, x) \leq v_{x}(\omega, t, x) \leq v(\omega, t, x), & \text { if } V_{0}(\omega, x)=\left(1-\partial_{x x}^{2}\right) u_{0}(\omega, x)>0, \\ v(\omega, t, x) \leq v_{x}(\omega, t, x) \leq-v(\omega, t, x), & \text { if } V_{0}(\omega, x)=\left(1-\partial_{x x}^{2}\right) u_{0}(\omega, x)<0 .\end{cases}
$$

Notice that

$$
\left\{V_{0}(\omega, x)>0\right\} \bigcap\left\{V_{0}(\omega, x)<0\right\}=\emptyset .
$$

Combining (5.14) and (5.15) yields

$$
\mathbb{P}\left\{\left|v_{x}(\omega, t, x)\right| \leq|v(\omega, t, x)|, \forall(t, x) \in\left[0, \tau^{*}\right) \times \mathbb{T}\right\} \geq p+q .
$$

In view of $H^{1} \hookrightarrow L^{\infty},(5.3)$ and (5.16), we arrive at

$$
\mathbb{P}\left\{\left\|v_{x}(\omega, t)\right\|_{L^{\infty}} \leq\|v(\omega, t)\|_{L^{\infty}} \lesssim\|v(\omega, t)\|_{H^{1}}=\left\|u_{0}\right\|_{H^{1}}, \forall t \in\left[0, \tau^{*}\right)\right\} \geq p+q .
$$

Via (5.1), we obtain the desired estimate.

Proof for Theorem 2.4 Let $\left(u, \tau^{*}\right)$ be the maximal pathwise solution to (1.8). Then Lemma 5.2 implies that

$$
\mathbb{P}\left\{\|u\|_{W^{1, \infty}} \lesssim 2 \beta(\omega, t)\left\|u_{0}\right\|_{H^{1}}, \forall t \in\left[0, \tau^{*}\right)\right\} \geq p+q .
$$

It follows from (i) in Lemma A.7 that $\sup _{t>0} \beta(\omega, t)<\infty \mathbb{P}-$ a.s. Then we can infer from (2.7) that $\mathbb{P}\left\{\tau^{*}=\infty\right\} \geq p+q$. That is to say, $\mathbb{P}\{u$ exists globally $\} \geq p+q$.

Acknowledgements Open Access funding provided by Projekt DEAL. The authors would like to express their great gratitude to the anonymous referees for their valuable suggestions, which have led to a meaningful improvement of this paper.

Open Access This article is licensed under a Creative Commons Attribution 4.0 International License, which permits use, sharing, adaptation, distribution and reproduction in any medium or format, as long as you give appropriate credit to the original author(s) and the source, provide a link to the Creative Commons licence, and indicate if changes were made. The images or other third party material in this article are included in the article's Creative Commons licence, unless indicated otherwise in a credit line to the material. If material is not included in the article's Creative Commons licence and your intended use is not permitted by statutory regulation or exceeds the permitted use, you will need to obtain permission directly from the copyright holder. To view a copy of this licence, visit http://creativecommons.org/licenses/by/4.0/.

\section{A Auxiliary Results}

In this section we formulate and prove some estimates employed in above proofs. We first recall the Friedrichs mollifier $J_{\varepsilon}$ defined as

$$
J_{\varepsilon} f(x)=j_{\varepsilon} * f(x) .
$$

Here $*$ stands for the convolution, $j_{\varepsilon}(x)=\sum_{k \in \mathbb{Z}} \widehat{j}(\varepsilon k) \mathrm{e}^{\mathrm{i} x k}$ and $j(x)$ is a Schwartz function satisfying $0 \leq \widehat{j}(\xi) \leq 1$ for all the $\xi \in \mathbb{R}$ and $j(\xi)=1$ for any $\xi \in[-1,1]$. We also need another mollifier $T_{\varepsilon}$ on $\mathbb{T}^{d}$ with $d \geq 1$ defined as

$$
T_{\varepsilon} f(x):=\left(1-\varepsilon^{2} \Delta\right)^{-1} f(x)=\sum_{k \in \mathbb{Z}^{d}}\left(1+\varepsilon^{2}|k|^{2}\right)^{-1} \widehat{f}(k) \mathrm{e}^{\mathrm{i} x \cdot k}, \quad \varepsilon \in(0,1) .
$$


From the construction, we see that $J_{\varepsilon}$ and $T_{\varepsilon}$ enjoy the following estimates, see $[58,59]$ for example,

$$
\begin{gathered}
{\left[D^{s}, J_{\varepsilon}\right]=\left[D^{s}, T_{\varepsilon}\right]=0,} \\
\left(J_{\varepsilon} f, g\right)_{L^{2}}\left(f, J_{\varepsilon} g\right)_{L^{2}},\left(T_{\varepsilon} f, g\right)_{L^{2}}=\left(f, T_{\varepsilon} g\right)_{L^{2}}, \\
\left\|J_{\varepsilon} u\right\|_{H^{s}},\left\|T_{\varepsilon} u\right\|_{H^{s}} \leq\|u\|_{H^{s}} .
\end{gathered}
$$

Lemma A.1 ( [59]) Let $d \geq 1$ and $f, g: \mathbb{T}^{d} \rightarrow \mathbb{R}^{d}$ such that $g \in W^{1, \infty}$ and $f \in L^{2}$. Then for some $C>0$,

$$
\left\|\left[T_{\varepsilon},(g \cdot \nabla)\right] f\right\|_{L^{2}} \leq C\|\nabla g\|_{L^{\infty}}\|f\|_{L^{2}} .
$$

Lemma A.2 ( [43]) If $f \in H^{s} \cap W^{1, \infty}, g \in H^{s-1} \bigcap L^{\infty}$ for $s>0$, then

$$
\left\|\left[D^{s}, f\right] g\right\|_{L^{2}} \leq C_{S}\left(\left\|D^{s} f\right\|_{L^{2}}\|g\|_{L^{\infty}}+\left\|\partial_{x} f\right\|_{L^{\infty}}\left\|D^{s-1} g\right\|_{L^{2}}\right) .
$$

Lemma A.3 ( [43]) Let $s>0$, for all $f, g \in H^{s} \cap L^{\infty}$,

$$
\|f g\|_{H^{s}} \leq C_{s}\left(\|f\|_{H^{s}}\|g\|_{L^{\infty}}+\|f\|_{L^{\infty}}\|g\|_{H^{s}}\right) .
$$

Lemma A.4 (Proposition 4.2, [64]) If $\rho>3 / 2$ and $0 \leq \eta+1 \leq \rho$, then for some $c>0$,

$$
\left\|\left[D^{\eta} \partial_{x}, f\right] v\right\|_{L^{2}} \leq c\|f\|_{H^{\rho}}\|v\|_{H^{\eta}}, \quad \forall f \in H^{\rho}, v \in H^{\eta} .
$$

Lemma A.5 For the $F(\cdot)$ defined in (1.6) with $F_{3}(u)$ disappearing for $k=1$, we have that for all $k \geq 1$,

$$
\begin{aligned}
\|F(v)\|_{H^{s}} & \lesssim\|v\|_{W^{1, \infty}}^{k}\|v\|_{H^{s}}, \quad s>3 / 2, \\
\|F(u)-F(v)\|_{H^{s}} & \lesssim\left(\|u\|_{H^{s}}+\|v\|_{H^{s}}\right)^{k}\|u-v\|_{H^{s}}, \quad s>3 / 2, \\
\|F(u)-F(v)\|_{H^{s}} & \lesssim\left(\|u\|_{H^{s+1}}+\|v\|_{H^{s+1}}\right)^{k}\|u-v\|_{H^{s}}, \quad 1 / 2<s \leq 3 / 2
\end{aligned}
$$

Proof Since $s>3 / 2$, the desired result follows from Lemma A.3 immediately. We omit the details and we refer to [63] for the $\mathrm{CH}$ case.

Lemma A.6 ( $[34,63])$ Let $\sigma, \alpha \in \mathbb{R}$. If $\lambda \in \mathbb{Z}^{+}$and $\lambda \gg 1$, then

$$
\|\sin (\lambda x-\alpha)\|_{H^{\sigma}}=\|\cos (\lambda x-\alpha)\|_{H^{\sigma}} \approx \lambda^{\sigma} .
$$

Lemma A.7 Let Assumption $A_{3}$ hold true and let $a(t) \in C([0, \infty))$ be a bounded function. For

$$
X=\mathrm{e}^{\int_{0}^{t} b\left(t^{\prime}\right) \mathrm{d} W_{t^{\prime}}+\int_{0}^{t} a\left(t^{\prime}\right)-\frac{b^{2}\left(t^{\prime}\right)}{2} \mathrm{~d} t^{\prime}},
$$

we have the following properties:

(i) Let $\phi(t):=\int_{0}^{t} b^{2}\left(t^{\prime}\right) \mathrm{d} t^{\prime}$ and $\phi^{-1}(t)$ be the inverse function of $\phi$. If

$$
\limsup _{t \rightarrow \infty} \frac{1}{\sqrt{2 t \log \log t}}\left(\int_{0}^{\phi^{-1}(t)} a\left(t^{\prime}\right) \mathrm{d} t^{\prime}-\frac{t}{2}\right)<-1,
$$

then

$$
\lim _{t \rightarrow \infty} X(t)=0 \mathbb{P}-\text { a.s. }
$$

If

$$
\liminf _{t \rightarrow \infty} \frac{1}{\sqrt{2 t \log \log t}}\left(\int_{0}^{\phi^{-1}(t)} a\left(t^{\prime}\right) \mathrm{d} t^{\prime}-\frac{t}{2}\right)>1,
$$


then

$$
\lim _{t \rightarrow \infty} X(t)=+\infty \mathbb{P}-\text { a.s. }
$$

(ii) Let $a(t)=\lambda b^{2}(t)$ with $\lambda<\frac{1}{2}$ and $\tau_{R}=\inf \{t \geq 0: X(t)>R\}$ with $R>1$, then

$$
\mathbb{P}\left(\tau_{R}=\infty\right) \geq 1-\left(\frac{1}{R}\right)^{1-2 \lambda} .
$$

Proof Let $Q(t)=\int_{0}^{t} b\left(t^{\prime}\right) \mathrm{d} W_{t^{\prime}}+\int_{0}^{t} a\left(t^{\prime}\right)-\frac{b^{2}\left(t^{\prime}\right)}{2} \mathrm{~d} t^{\prime}$. Since $\phi:[0, \infty) \ni t \mapsto \phi(t) \in$ $[0, \infty)$ is one-to-one (since $\phi$ is strictly increasing) and onto (by Assumption $\left.A_{3}\right), \phi^{-1}(t)$ is well defined and $\lim _{t \rightarrow \infty} Q(t)=\lim _{t \rightarrow \infty} Q\left(\phi^{-1}(t)\right) \mathbb{P}-$ a.s. Direct computation (see [1, Exercise 7.7 and Theorem 8.2] for example) shows that

$$
B_{t}:=\int_{0}^{\phi^{-1}(t)} b\left(t^{\prime}\right) \mathrm{d} W_{t^{\prime}}
$$

is itself a Brownian motion. Then we use the law of the iterated logarithm to get

$$
\limsup _{t \rightarrow \infty} \frac{B_{t}}{\sqrt{2 t \log \log t}}=1, \liminf _{t \rightarrow \infty} \frac{B_{t}}{\sqrt{2 t \log \log t}}=-1 \mathbb{P}-\text { a.s. }
$$

Therefore (A.4) implies

$$
\lim _{t \rightarrow \infty} Q\left(\phi^{-1}(t)\right) \leq \limsup _{t \rightarrow \infty} \sqrt{2 t \log \log t}\left(\frac{B_{t}}{\sqrt{2 t \log \log t}}+\frac{\int_{0}^{\phi^{-1}(t)} a\left(t^{\prime}\right) \mathrm{d} t^{\prime}-\frac{t}{2}}{\sqrt{2 t \log \log t}}\right)=-\infty \mathbb{P}-\text { a.s. }
$$

which leads to

$$
\lim _{t \rightarrow \infty} X(t)=\mathrm{e}^{\lim _{t \rightarrow \infty} Q\left(\phi^{-1}(t)\right)}=0 \mathbb{P}-\text { a.s. }
$$

Hence we obtain (A.5). If (A.6) holds true, then

$$
\lim _{t \rightarrow \infty} Q\left(\phi^{-1}(t)\right) \geq \liminf _{t \rightarrow \infty} \sqrt{2 t \log \log t}\left(\frac{B_{t}}{\sqrt{2 t \log \log t}}+\frac{\int_{0}^{\phi^{-1}(t)} a\left(t^{\prime}\right) \mathrm{d} t^{\prime}-\frac{t}{2}}{\sqrt{2 t \log \log t}}\right)=+\infty \mathbb{P}-\text { a.s. }
$$

which gives (A.7). Now we consider (A.8) and exploit [29]. It is easy to see that when $a(t)=\lambda b^{2}(t), X$ is the unique global solution to the problem

$$
\mathrm{d} X=\lambda b^{2}(t) X \mathrm{~d} t+b(t) X \mathrm{~d} W, \quad X(0)=1 .
$$

We apply the Itô formula to $X^{p}$ with $p>0$ to obtain that

$$
\mathrm{d} X^{p}=\left(p \lambda b^{2}(t)+\frac{b^{2}(t) p(p-1)}{2}\right) X^{p} \mathrm{~d} t+p b(t) X^{p} \mathrm{~d} W .
$$

Particularly, if $p=1-2 \lambda$, then

$$
\mathrm{d} X^{1-2 \lambda}=(1-2 \lambda) b(t) X^{1-2 \lambda} \mathrm{d} W
$$

which means $\mathbb{E} X^{1-2 \lambda}\left(t \wedge \tau_{R}\right)=1$. Hence by the definition of $\tau_{R}$, continuity of measures and the Chebyshev inequality, we have

$$
\begin{aligned}
\mathbb{P}\left\{\tau_{R}=\infty\right\} & =\lim _{N \rightarrow \infty} \mathbb{P}\left(\tau_{R}>N\right) \\
& =\lim _{N \rightarrow \infty} \mathbb{P}\left(X^{1-2 \lambda}\left(N \wedge \tau_{R}\right)<R^{1-2 \lambda}\right)
\end{aligned}
$$




$$
\geq \lim _{N \rightarrow \infty}\left(1-\frac{\mathbb{E} X^{1-2 \lambda}\left(N \wedge \tau_{R}\right)}{R^{1-2 \lambda}}\right)=1-\frac{1}{R^{1-2 \lambda}},
$$

which is (A.8).

\section{References}

1. Baldi, P.: Stochastic Calculus. Universitext. Springer, Cham (2017)

2. Bona, J.L., Tzvetkov, N.: Sharp well-posedness results for the BBM equation. Discrete Contin. Dyn. Syst. 23(4), 1241-1252 (2009)

3. Breit, D., Feireisl, E., Hofmanová, M.: Local strong solutions to the stochastic compressible Navier-Stokes system. Comm. Partial Differ. Equ. 43(2), 313-345 (2018)

4. Breit, D., Feireisl, E., Hofmanová, M.: Stochastically forced compressible fluid flows. De Gruyter Series in Applied and Numerical Mathematics, vol. 3. De Gruyter, Berlin (2018)

5. Breit, D., Hofmanová, M.: Stochastic Navier-Stokes equations for compressible fluids. Indiana Univ. Math. J. 65(4), 1183-1250 (2016)

6. Bressan, A., Constantin, A.: Global conservative solutions of the Camassa-Holm equation. Arch. Ration. Mech. Anal. 183(2), 215-239 (2007)

7. Bressan, A., Constantin, A.: Global dissipative solutions of the Camassa-Holm equation. Anal. Appl. (Singap.) 5(1), 1-27 (2007)

8. Camassa, R., Holm, D.D.: An integrable shallow water equation with peaked solitons. Phys. Rev. Lett. 71(11), 1661-1664 (1993)

9. Chen, D., Li, Y., Yan, W.: On the Cauchy problem for a generalized Camassa-Holm equation. Discrete Contin. Dyn. Syst. 35(3), 871-889 (2015)

10. Chen, Y., Gao, H.: Well-posedness and large deviations of the stochastic modified Camassa-Holm equation. Potential Anal. 45(2), 331-354 (2016)

11. Chen, Y., Gao, H., Guo, B.: Well-posedness for stochastic Camassa-Holm equation. J. Differ. Equ. 253(8), 2353-2379 (2012)

12. Constantin, A.: On the blow-up of solutions of a periodic shallow water equation. J. Nonlinear Sci. 10(3), 391-399 (2000)

13. Constantin, A.: The trajectories of particles in Stokes waves. Invent. Math. 166(3), 523-535 (2006)

14. Constantin, A., Escher, J.: Wave breaking for nonlinear nonlocal shallow water equations. Acta Math. 181(2), 229-243 (1998)

15. Constantin, A., Escher, J.: Well-posedness, global existence, and blowup phenomena for a periodic quasilinear hyperbolic equation. Comm. Pure Appl. Math. 51(5), 475-504 (1998)

16. Constantin, A., Escher, J.: Particle trajectories in solitary water waves. Bull. Am. Math. Soc. (N.S.) 44(3), 423-431 (2007)

17. Constantin, A., Escher, J.: Analyticity of periodic traveling free surface water waves with vorticity. Ann. Math. (2) 173(1), 559-568 (2011)

18. Crisan, D., Flandoli, F., Holm, D.D.: Solution properties of a 3D stochastic Euler fluid equation. J. Nonlinear Sci. 29(3), 813-870 (2019)

19. Da Prato, G., Zabczyk, J.: Stochastic Equations in Infinite Dimensions, volume 152 of Encyclopedia of Mathematics and its Applications. Cambridge University Press, Cambridge, second edition (2014)

20. Debussche, A., Glatt-Holtz, N.E., Temam, R.: Local martingale and pathwise solutions for an abstract fluids model. Phys. D 240(14-15), 1123-1144 (2011)

21. Fedrizzi, E., Neves, W., Olivera, C.: On a class of stochastic transport equations for $L_{\text {loc }}^{2}$ vector fields. Ann. Sc. Norm. Super. Pisa Cl. Sci. (5) 18(2), 397-419 (2018)

22. Flandoli, F.: An introduction to 3D stochastic fluid dynamics. In: SPDE in Hydrodynamic: Recent Progress and Prospects vol. 1942 (pp. 51-150). Springer, Berlin (2008)

23. Flandoli, F., Gubinelli, M., Priola, E.: Well-posedness of the transport equation by stochastic perturbation. Invent. Math. 180(1), 1-53 (2010)

24. Fuchssteiner, B., Fokas, A. S.: Symplectic structures, their Bäcklund transformations and hereditary symmetries. Phys. D 4(1): 47-66 (1981/82)

25. Gawarecki, L., Mandrekar, V.: Stochastic differential equations in infinite dimensions with applications to stochastic partial differential equations. Probability and its Applications (New York). Springer, Heidelberg (2011)

26. Geng, X., Xue, B.: An extension of integrable peakon equations with cubic nonlinearity. Nonlinearity 22(8), 1847-1856 (2009) 
27. Gess, B., Souganidis, P.E.: Long-time behavior, invariant measures, and regularizing effects for stochastic scalar conservation laws. Comm. Pure Appl. Math. 70(8), 1562-1597 (2017)

28. Glatt-Holtz, N., Ziane, M.: Strong pathwise solutions of the stochastic Navier-Stokes system. Adv. Differ. Equ. 14(5-6), 567-600 (2009)

29. Glatt-Holtz, N.E., Vicol, V.C.: Local and global existence of smooth solutions for the stochastic Euler equations with multiplicative noise. Ann. Probab. 42(1), 80-145 (2014)

30. Gyöngy, I., Krylov, N.: Existence of strong solutions for Itô's stochastic equations via approximations. Probab. Theory Related Fields 105(2), 143-158 (1996)

31. Henry, D.: Geometric Theory of Semilinear Parabolic Equations, p. iv+348. Springer, Berlin (1981)

32. Himonas, A.A., Holliman, C.: The Cauchy problem for a generalized Camassa-Holm equation. Adv. Differ. Equ. 19(1-2), 161-200 (2014)

33. Himonas, A.A., Kenig, C.: Non-uniform dependence on initial data for the $\mathrm{CH}$ equation on the line. Differ. Integral Equ. 22(3-4), 201-224 (2009)

34. Himonas, A.A., Kenig, C., Misiołek, G.: Non-uniform dependence for the periodic CH equation. Comm. Partial Differ. Equ. 35(6), 1145-1162 (2010)

35. Himonas, A.A., Misiołek, G.: Non-uniform dependence on initial data of solutions to the Euler equations of hydrodynamics. Comm. Math. Phys. 296(1), 285-301 (2010)

36. Hofmanová, M.: Degenerate parabolic stochastic partial differential equations. Stochastic Process. Appl. 123(12), 4294-4336 (2013)

37. Holden, H., Raynaud, X.: Global conservative solutions of the Camassa-Holm equation-a Lagrangian point of view. Comm. Partial Differ. Equ. 32(10-12), 1511-1549 (2007)

38. Holden, H., Raynaud, X.: Dissipative solutions for the Camassa-Holm equation. Discrete Contin. Dyn. Syst. 24(4), 1047-1112 (2009)

39. Hone, A.N.W., Lundmark, H., Szmigielski, J.: Explicit multipeakon solutions of Novikov's cubically nonlinear integrable Camassa-Holm type equation. Dyn. Partial Differ. Equ. 6(3), 253-289 (2009)

40. Kallianpur, G., Xiong, J., Stochastic differential equations in infinite-dimensional spaces. 26:vi+342, : Expanded version of the lectures delivered as part of the 1993 Barrett Lectures at the University of Tennessee, Knoxville, TN, March 25-27, 1993. With a foreword by Balram S, Rajput and Jan Rosinski (1995)

41. Karczewska, A.: Stochastic integral with respect to cylindrical Wiener process. Ann. Univ. Mariae CurieSkłodowska Sect. A 52(2), 79-93 (1998)

42. Kato, T.: The Cauchy problem for quasi-linear symmetric hyperbolic systems. Arch. Rational Mech. Anal. 58(3), 181-205 (1975)

43. Kato, T., Ponce, G.: Commutator estimates and the Euler and Navier-Stokes equations. Comm. Pure Appl. Math. 41(7), 891-907 (1988)

44. Kenig, C.E., Ponce, G., Vega, L.: Well-posedness and scattering results for the generalized Korteweg-de Vries equation via the contraction principle. Comm. Pure Appl. Math. 46(4), 527-620 (1993)

45. Kenig, C.E., Ponce, G., Vega, L.: On the ill-posedness of some canonical dispersive equations. Duke Math. J. 106(3), 617-633 (2001)

46. Kim, J.U.: On the Cauchy problem for the transport equation with random noise. J. Funct. Anal. 259(12), 3328-3359 (2010)

47. Koch, H., Tzvetkov, N.: Nonlinear wave interactions for the Benjamin-Ono equation. Int. Math. Res. Not. 30, 1833-1847 (2005)

48. Kröker, I., Rohde, C.: Finite volume schemes for hyperbolic balance laws with multiplicative noise. Appl. Numer. Math. 62(4), 441-456 (2012)

49. Lenells, J., Wunsch, M.: On the weakly dissipative Camassa-Holm, Degasperis-Procesi, and Novikov equations. J. Differ. Equ. 255(3), 441-448 (2013)

50. Lions, P.-L., Perthame, B., Souganidis, P.E.: Scalar conservation laws with rough (stochastic) fluxes. Stoch. Partial Differ. Equ. Anal. Comput. 1(4), 664-686 (2013)

51. Lions, P.-L., Perthame, B., Souganidis, P.E.: Scalar conservation laws with rough (stochastic) fluxes: the spatially dependent case. Stoch. Partial Differ. Equ. Anal. Comput. 2(4), 517-538 (2014)

52. McKean, H.P.: Breakdown of a shallow water equation. Asian J. Math., 2(4), 867-874 (1998). Mikio Sato: a great Japanese mathematician of the twentieth century

53. Mollinedo, D.A.C., Olivera, C.: Well-posedness of the stochastic transport equation with unbounded drift. Bull. Braz. Math. Soc. (N.S.) 48(4), 663-677 (2017)

54. Neves, W., Olivera, C.: Wellposedness for stochastic continuity equations with Ladyzhenskaya-ProdiSerrin condition. NoDEA Nonlinear Differ. Equ. Appl. 22(5), 1247-1258 (2015)

55. Novikov, V.: Generalizations of the Camassa-Holm equation. J. Phys. A 42(34), 342002 (2009)

56. Prévôt, C., Röckner, M.: A Concise Course on Stochastic Partial Differential Equations. Lecture Notes in Mathematics. Springer, Berlin (2007) 
57. Röckner, M., Zhu, R., Zhu, X.: Local existence and non-explosion of solutions for stochastic fractional partial differential equations driven by multiplicative noise. Stochastic Process. Appl. 124(5), 1974-2002 (2014)

58. Tang, H.: On the pathwise solutions to the Camassa-Holm equation with multiplicative noise. SIAM J. Math. Anal. 50(1), 1322-1366 (2018)

59. Tang, H.: Noise effects on dependence on initial data and blow-up for stochastic Euler-Poincaré equations. arXiv:2002.08719 (2020)

60. Tang, H., Liu, Z.: Continuous properties of the solution map for the Euler equations. J. Math. Phys. 55(3), 031504 (2014)

61. Tang, H., Liu, Z.: Well-posedness of the modified Camassa-Holm equation in Besov spaces. Z. Angew. Math. Phys. 66(4), 1559-1580 (2015)

62. Tang, H., Shi, S., Liu, Z.: The dependences on initial data for the b-family equation in critical Besov space. Monatsh. Math. 177(3), 471-492 (2015)

63. Tang, H., Zhao, Y., Liu, Z.: A note on the solution map for the periodic Camassa-Holm equation. Appl. Anal. 93(8), 1745-1760 (2014)

64. Taylor, M.: Commutator estimates. Proc. Am. Math. Soc. 131(5), 1501-1507 (2003)

65. Wu, S., Yin, Z.: Global existence and blow-up phenomena for the weakly dissipative Camassa-Holm equation. J. Differ. Equ. 246(11), 4309-4321 (2009)

66. Zhao, Y., Li, Y., Yan, W.: Local well-posedness and persistence property for the generalized Novikov equation. Discrete Contin. Dyn. Syst. 34(2), 803-820 (2014)

67. Zhou, S., Mu, C.: The properties of solutions for a generalized $b$-family equation with peakons. J. Nonlinear Sci. 23(5), 863-889 (2013)

Publisher's Note Springer Nature remains neutral with regard to jurisdictional claims in published maps and institutional affiliations. 